\title{
Heat-diffusion and Poisson integrals for Laguerre and special Hermite expansions on weighted $L^{p}$ spaces
}

by

ADAM NOWAK (Wrocław)

\begin{abstract}
We investigate heat-diffusion and Poisson integrals associated with Laguerre and special Hermite expansions on weighted $L^{p}$ spaces with $A_{p}$ weights.
\end{abstract}

1. Introduction. Heat-diffusion and Poisson integrals for Laguerre polynomial expansions were first studied by Muckenhoupt [Mu1]. Then Stempak [St], motivated by Muckenhoupt's paper, considered one-dimensional Laguerre expansions with respect to different systems of Laguerre functions. Multi-dimensional Hermite function expansions in weighted $L^{p}$ setting have recently been considered by Stempak and Torrea [StTo]. Our aim is to go further and discuss multi-dimensional Laguerre and special Hermite function expansions in weighted $L^{p}$ setting. We note that some aspects of weighted $L^{p}$ theory for special Hermite and certain one-dimensional Laguerre expansions have been recently treated also by Kerman and Thangavelu $[\mathrm{KeTh}]$.

In this paper we consider expansions with respect to special Hermite functions and two different systems of Laguerre functions. We define (pointwise) corresponding heat-diffusion and Poisson integrals in weighted $L^{p}$ setting with weights from Muckenhoupt's $A_{p}$ class, $1 \leq p<\infty$. Then we investigate their smoothness, boundary behavior and mapping properties. In particular we show that the associated maximal operators are dominated, up to a constant, by the Hardy-Littlewood maximal function or by the strong maximal function. We follow closely the technique used in [StTo] for ordinary multi-dimensional Hermite expansions and the corresponding integrals.

2000 Mathematics Subject Classification: Primary 42C10.

Key words and phrases: special Hermite expansions, Laguerre expansions, heatdiffusion semigroup, Poisson semigroup, $A_{p}$ weights.

Research supported partially by Research Training Network "Harmonic Analysis and Related Problems", contract HPRN-CT-2001-00273-HARP. 
The paper is organized as follows. Section 2 contains some basic facts and notation needed in what follows. In Sections 3, 4 and 5 we treat in order special Hermite expansions, Laguerre expansions based on the system $\left\{\ell_{k}^{\alpha}\right\}$, and Laguerre expansions with respect to the system $\left\{\varphi_{k}^{\alpha}\right\}$. Main results of these sections are contained in Theorems 3.10, 4.6 and 5.5. Finally, in Section 6 we give some remarks on the connection between the Hermite and Laguerre cases, including an extension of the transference studied in [Di] and [GIT].

2. Preliminaries. Throughout the paper we use standard notation. $\mathbb{N}=\{0,1, \ldots\}$ denotes the set of natural numbers, $\mathbb{R}, \mathbb{C}$ real and complex numbers, respectively. Given $x \in \mathbb{R}^{n}$ or $x \in \mathbb{C}^{n}$, we denote by $|x|$ its Euclidean norm. For a multi-index $\alpha=\left(\alpha_{1}, \ldots, \alpha_{n}\right),|\alpha|$ stands for its length, i.e. $|\alpha|=\sum_{i=1}^{n} \alpha_{i}$. If $x \in \mathbb{R}^{n}$ then $x^{\alpha}=x_{1}^{\alpha_{1}} \ldots x_{n}^{\alpha_{n}}$. The function classes $C_{\mathrm{c}}^{\infty}, C_{0}, L^{p}, L^{p}(\omega), A_{p}$ are defined in a standard manner and will consist of functions defined on $\mathbb{C}^{n}$ or $\mathbb{R}_{+}^{d}=(0, \infty)^{d}$. For $p \in[1, \infty)$ the norm in the weighted space $L^{p}(\omega)$ is denoted by $\|\cdot\|_{L^{p}(\omega)}$. If $\omega \equiv 1$ the notation is simplified to $\|\cdot\|_{p}$. We write $p^{\prime}$ for the conjugate of $p\left(\right.$ i.e. $\left.1 / p+1 / p^{\prime}=1\right)$.

We adopt the convention that constants may change their value from one use to the next. The notation $c=c(\alpha, \beta, \gamma)$ means that $c$ is a constant depending only on $\alpha, \beta, \gamma$. Constants are always strictly positive and finite.

Given $\alpha>-1$, the one-dimensional Laguerre polynomials of type $\alpha$ are

$$
L_{k}^{\alpha}(x)=\frac{1}{k !} e^{x} x^{-\alpha} \frac{d^{k}}{d x^{k}}\left(e^{-x} x^{k+\alpha}\right), \quad k \in \mathbb{N}, x>0 .
$$

Note that each $L_{k}^{\alpha}$ is a polynomial of degree $k$. Given a multi-index $\alpha=$ $\left(\alpha_{1}, \ldots, \alpha_{d}\right), \alpha \in(-1, \infty)^{d}$, the $d$-dimensional Laguerre polynomials of type $\alpha$ are tensor products of the one-dimensional Laguerre polynomials, i.e.

$$
L_{k}^{\alpha}(x)=\prod_{i=1}^{d} L_{k_{i}}^{\alpha_{i}}\left(x_{i}\right), \quad k \in \mathbb{N}^{d}, x \in \mathbb{R}_{+}^{d} .
$$

Similarly, multi-dimensional Hermite polynomials $H_{k}, k \in \mathbb{N}^{d}$, are tensor products of one-dimensional Hermite polynomials defined by

$$
H_{0}(x)=1, \quad H_{k}(x)=e^{x^{2}} \frac{d^{k}}{d x^{k}} e^{-x^{2}}, \quad k \geq 1, x \in \mathbb{R} .
$$

The Hermite functions $h_{k}$ in $\mathbb{R}^{d}$ are given by

$$
h_{k}(x)=c_{k} H_{k}(x) \exp \left(-|x|^{2} / 2\right), \quad x \in \mathbb{R}^{d},
$$

where $k=\left(k_{1}, \ldots, k_{d}\right) \in \mathbb{N}^{d}$ and $c_{k}=\prod_{i=1}^{d}\left(\sqrt{\pi} 2^{k_{i}} \Gamma\left(k_{i}+1\right)\right)^{-1 / 2}$. Each $h_{k}$ is an eigenfunction of the $d$-dimensional Hermite operator (harmonic oscillator)

$$
L=-\Delta+|x|^{2},
$$


the corresponding eigenvalue being $2|k|+d$. The operator $L$ is positive and symmetric in $L^{2}\left(\mathbb{R}^{d}\right)$. Moreover, the system $\left\{h_{k}: k \in \mathbb{N}^{d}\right\}$ is an orthonormal basis in $L^{2}\left(\mathbb{R}^{d}\right)$.

3. Special Hermite expansions. In this section we study heat-diffusion and Poisson semigroups associated with the special Hermite operator $\widetilde{\Delta}$, usually called the twisted Laplacian. The operator $\widetilde{\Delta}$ is closely related to the sublaplacian on the Heisenberg group $\mathbb{H}^{n}$, and special Hermite expansions play an important role in a better understanding of some problems on $\mathbb{H}^{n}$ (see [Th2]).

Let $n \geq 1$ and $(x, y) \in \mathbb{R}^{n} \times \mathbb{R}^{n} \simeq \mathbb{C}^{n}$. Then we have

$$
\widetilde{\Delta}=-\Delta_{x}-\Delta_{y}+\frac{1}{4}\left(|x|^{2}+|y|^{2}\right)-i \sum_{j=1}^{n}\left(x_{j} \frac{\partial}{\partial y_{j}}-y_{j} \frac{\partial}{\partial x_{j}}\right),
$$

where $\Delta_{x}=\sum_{j=1}^{n} \partial_{x_{j}}^{2}, \Delta_{y}=\sum_{j=1}^{n} \partial_{y_{j}}^{2}$ are the standard Laplacians on $\mathbb{R}^{n}$. The set of eigenfunctions of this operator contains special Hermite functions $\Phi_{\alpha, \beta}\left(\alpha, \beta \in \mathbb{N}^{n}\right)$. These form a complete orthonormal system in $L^{2}\left(\mathbb{C}^{n}\right)$ and are given by the following Fourier-Wigner transform of the usual Hermite functions $h_{\alpha}$ and $h_{\beta}$ :

$$
\Phi_{\alpha, \beta}(z)=(2 \pi)^{-n / 2} \int_{\mathbb{R}^{n}} e^{i x \cdot \xi} h_{\alpha}\left(\xi+\frac{y}{2}\right) h_{\beta}\left(\xi-\frac{y}{2}\right) d \xi, \quad z=x+i y \in \mathbb{C}^{n} .
$$

The spectrum of $\widetilde{\Delta}$ is discrete and equals $\{2 k+n: k \in \mathbb{N}\}$. Since we have $\widetilde{\Delta} \Phi_{\alpha, \beta}=(2|\beta|+n) \Phi_{\alpha, \beta}$, the eigenspace corresponding to the eigenvalue $2 k+n$ is infinite-dimensional and spanned by $\left\{\Phi_{\alpha, \beta}:|\beta|=k\right\}$. For a function $f \in L^{2}\left(\mathbb{C}^{n}\right)$ the series

$$
f=\sum_{\alpha, \beta}\left\langle f, \Phi_{\alpha, \beta}\right\rangle \Phi_{\alpha, \beta}
$$

is convergent in $L^{2}\left(\mathbb{C}^{n}\right)$ and is called the special Hermite expansion of $f$. Denote by $Q_{k}$ the spectral projection operator on the eigenspace corresponding to the $k$ th eigenvalue $2 k+n$. Then the series (1) may be written in a compact form

$$
f=\sum_{k=0}^{\infty} Q_{k} f
$$

Given functions $f, g \in L^{2}\left(\mathbb{C}^{n}\right)$, their twisted convolution is defined as

$$
f \times g(z)=\int_{\mathbb{C}^{n}} f(z-u) g(u) \exp \left(\frac{1}{2} i \operatorname{Im}\langle z, u\rangle\right) d u,
$$

where $\langle z, u\rangle=\sum_{j=1}^{n} z_{j} \bar{u}_{j}$. The above product turns $L^{1}\left(\mathbb{C}^{n}\right)$ into a (noncommutative) Banach algebra. 
The spectral projections $Q_{k}$ are then expressed as

$$
Q_{k} f=f \times \phi_{k}^{n-1},
$$

$\phi_{k}^{n-1}$ being the Laguerre functions defined by

$$
\phi_{k}^{n-1}(z)=(2 \pi)^{-n} L_{k}^{n-1}\left(|z|^{2} / 2\right) \exp \left(-|z|^{2} / 4\right), \quad z \in \mathbb{C}^{n} .
$$

Each $\phi_{k}^{n-1}$ is an eigenfunction of $\widetilde{\Delta}$ that corresponds to the eigenvalue $2 k+n$ and $\left\{\phi_{k}^{n-1}: k \in \mathbb{N}\right\}$ is an orthogonal (but incomplete) system in $L^{2}\left(\mathbb{C}^{n}\right)$. For all of the above and further facts regarding special Hermite expansions the reader is referred to the book of Thangavelu [Th1].

Let $m \geq 1$ and define

$$
\vartheta_{k}^{m-1}(z)= \begin{cases}(2 k+m)^{m-1}, & |z| \leq \sqrt{6(2 k+m)} \\ \exp \left(-\gamma|z|^{2}\right), & |z|>\sqrt{6(2 k+m)}\end{cases}
$$

We will make use of the estimate

$$
\left|\phi_{k}^{m-1}(z)\right| \leq c \vartheta_{k}^{m-1}
$$

which is a consequence of estimates for Laguerre functions due to Askey and Wainger $[\mathrm{AW}]$, compiled by Muckenhoupt [Mu2]. Here $c$ and $\gamma$ are independent of $k \in \mathbb{N}$ and $z \in \mathbb{C}^{n}$. A direct calculation using (2) shows that

$$
\left\|\phi_{k}^{n-1}\right\|_{p} \leq c(n)(2 k+n)^{2 n-1}, \quad 1 \leq p<\infty .
$$

This estimate may be generalized by adding a proper weight to the $L^{p}$ norm. In fact, we have the following

Proposition 3.1. Let $1 \leq p<\infty$ and $\omega \in A_{p}\left(\mathbb{C}^{n}\right)$. There exists a constant $c$ (independent of $k$ ) such that

$$
\left\|\phi_{k}^{n-1}\right\|_{L^{p}(\omega)} \leq c(2 k+n)^{2 n-1} .
$$

Proof. Denote by $B_{r}$ the ball $\left\{z \in \mathbb{C}^{n}:|z| \leq r\right\}$. Since the $A_{p}$ condition implies

$$
\frac{\omega\left(B_{r}\right)}{\omega\left(B_{1}\right)} \leq c\left(\frac{\left|B_{r}\right|}{\left|B_{1}\right|}\right)^{p}, \quad r \geq 1
$$

we have

$$
\omega\left(B_{r}\right) \leq c r^{2 n p}, \quad r \geq 1 .
$$

Let $\Gamma_{1}=B \sqrt{6(2 k+n)}$ and $\Gamma_{2}=\mathbb{C}^{n} \backslash \Gamma_{1}$. We decompose $\Gamma_{2}$ into disjoint "rings":

$$
\Gamma_{2}=\bigcup_{m=0}^{\infty} \Gamma_{2}^{m}, \quad \Gamma_{2}^{m}=\left\{z \in \mathbb{C}^{n}: 2^{m} \sqrt{6(2 k+n)}<|z| \leq 2^{m+1} \sqrt{6(2 k+n)}\right\} .
$$


Now, to estimate LHS in (3) we split the integration over $\mathbb{C}^{n}$ into integration over $\Gamma_{1}$ and over each of $\Gamma_{2}^{m}$. By (2) and (4) we obtain

$$
\begin{aligned}
\int_{\Gamma_{1}}\left|\phi_{k}^{n-1}(z)\right|^{p} d z & \leq c \int_{\Gamma_{1}}(2 k+n)^{(n-1) p} \omega(z) d z=c(2 k+n)^{(n-1) p} \omega\left(\Gamma_{1}\right) \\
& \leq c(2 k+n)^{(n-1) p}(\sqrt{6(2 k+n)})^{2 n p}=c(2 k+n)^{(2 n-1) p},
\end{aligned}
$$

and

$$
\begin{aligned}
\int_{\Gamma_{2}}\left|\phi_{k}^{n-1}(z)\right|^{p} d z & =\sum_{m=0}^{\infty} \int_{\Gamma_{2}^{m}}\left|\phi_{k}^{n-1}(z)\right|^{p} d z \\
& \leq c \sum_{m=0}^{\infty} \exp \left(-\gamma p 2^{2 m} 6(2 k+n)\right) \omega\left(B_{2^{m+1}} \sqrt{6(2 k+n)}\right) \\
& \leq c(2 k+n)^{n p} \sum_{m=0}^{\infty}\left(2^{2 m}\right)^{n p} \exp \left(-\gamma 2^{2 m}\right) \leq c(2 k+n)^{(2 n-1) p}
\end{aligned}
$$

In what follows we will make use of the following lemma (cf. [S, p. 198]).

LEMMA 3.2. Assume that $\Psi: \mathbb{C}^{n} \rightarrow[0, \infty)$ is radial, and (radially) decreasing, with $\int \Psi(z) d z=1$. Define $\Psi_{\varepsilon}(z)=\varepsilon^{-2 n} \Psi(z / \varepsilon), \varepsilon>0$. If $1 \leq p<\infty$ and $\omega \in A_{p}\left(\mathbb{C}^{n}\right)$ then

$$
\left\|f * \Psi_{\varepsilon}\right\|_{L^{p}(\omega)} \leq A\|f\|_{L^{p}(\omega)}, \quad f \in L^{p}(\omega),
$$

with $A$ independent of $\varepsilon$ and $\Psi$. Moreover, $A$ depends on $\omega$ only through the $A_{p}$ norm of $\omega$.

REMARK 3.3. Denote by $\mathcal{R}$ the class of functions $\Psi$ satisfying the assumptions of Lemma 3.2 (notice that if $\Psi$ belongs to $\mathcal{R}$ then so does $\Psi_{\varepsilon}$ ). Then

$$
M f(z)=\sup _{\Psi \in \mathcal{R}}|f| * \Psi(z), \quad z \in \mathbb{C}^{n},
$$

where $M$ denotes the (centered) Hardy-Littlewood maximal function in $\mathbb{R}^{2 n} \simeq \mathbb{C}^{n}$. See $[\mathrm{S}]$ for details.

Now we are in a position to estimate weighted $L^{p}$ norms of $Q_{k} f$.

Lemma 3.4. Let $1 \leq p<\infty$ and $\omega \in A_{p}$. Then

$$
\left\|Q_{k} f\right\|_{L^{p}(\omega)} \leq c(2 k+n)^{2 n-1}\|f\|_{L^{p}(\omega)}, \quad f \in L^{p}(\omega),
$$

with $c$ independent of $k \in \mathbb{N}$.

Proof. Observe that by (2),

$$
\left|Q_{k} f(z)\right|=\left|f \times \phi_{k}^{n-1}(z)\right| \leq|f| *\left|\phi_{k}^{n-1}\right|(z) \leq c|f| * \vartheta_{k}^{n-1}(z),
$$


and notice that $\vartheta_{k}^{n-1}$ is radial and (radially) decreasing. Thus Lemma 3.2 may be applied to the function $\vartheta_{k}^{n-1} /\left\|\vartheta_{k}^{n-1}\right\|_{1}$. As a result we obtain

$$
\left\||f| * \vartheta_{k}^{n-1}\right\|_{L^{p}(\omega)} \leq c\left\|\vartheta_{k}^{n-1}\right\|_{1}\|f\|_{L^{p}(\omega)} \leq c(2 k+n)^{2 n-1}\|f\|_{L^{p}(\omega)} .
$$

For the last inequality see the proof of Proposition 3.1.

Our next objective is to obtain a pointwise estimate of $Q_{k} f$.

Lemma 3.5. Let $1 \leq p<\infty, \omega \in A_{p}$ and $f \in L^{p}(\omega)$. Then

$$
\left|Q_{k} f(z)\right| \leq c(2 k+n)^{n-1}(\sqrt{6(2 k+n)}+|z|)^{2 n}\|f\|_{L^{p}(\omega)}, \quad z \in \mathbb{C}^{n},
$$

with $c$ independent of $k \in \mathbb{N}$.

Proof. Without loss of generality we assume $f \geq 0$. For a function $v$ on $\mathbb{C}^{n}$ we define

$$
\left(\tau_{\xi} v\right)(z)=v(z-\xi), \quad \check{v}(z)=v(-z), \quad z \in \mathbb{C}^{n} .
$$

As in the proof of Lemma 3.4 we have

$$
\left|Q_{k} f(z)\right| \leq c f * \vartheta_{k}^{n-1}(z)=c \int_{\mathbb{C}^{n}} f(u) \tau_{z} \check{\vartheta}_{k}^{n-1}(u) d u .
$$

To handle the integral above observe that

$$
\tau_{z} \check{\vartheta}_{k}^{n-1}(u) \leq \begin{cases}(2 k+n)^{n-1}, & |u| \leq \sqrt{6(2 k+n)}+|z|, \\ \exp \left(-\gamma(|u|-|z|)^{2}\right), & |u|>\sqrt{6(2 k+n)}+|z| .\end{cases}
$$

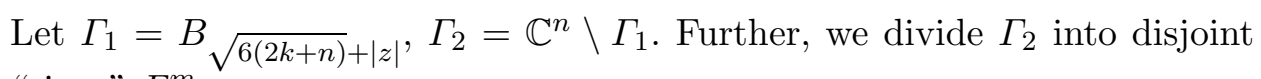
"rings" $\Gamma_{2}^{m}$ :

$$
\Gamma_{2}^{m}=\left\{u \in \mathbb{C}^{n}: 2^{m} \sqrt{6(2 k+n)}+|z|<|u| \leq 2^{m+1} \sqrt{6(2 k+n)}+|z|\right\} .
$$

Consider the case $1<p<\infty$. By Hölder's inequality we obtain

$$
\begin{aligned}
\int_{\mathbb{C}^{n}} f(u) \tau_{z} \check{\vartheta}_{k}^{n-1}(u) d u & \leq\|f\|_{L^{p}(\omega)}\left(\int_{\mathbb{C}^{n}}\left(\tau_{z} \check{\vartheta}_{k}^{n-1}(u)\right)^{p^{\prime}} \omega(u)^{-p^{\prime} / p} d u\right)^{1 / p^{\prime}} \\
& =\|f\|_{L^{p}(\omega)}\left\|\tau_{z} \check{\vartheta}_{k}^{n-1}\right\|_{L^{p^{\prime}}(\widetilde{\omega})} .
\end{aligned}
$$

Since $\widetilde{\omega}=\omega^{-p^{\prime} / p}$ belongs to $A_{p^{\prime}}$ (cf. [D]), similarly to the proof of Proposition 3.1 we get

$$
\left\|\tau_{z} \check{\vartheta}_{k}^{n-1}\right\|_{L^{p^{\prime}}(\widetilde{\omega})} \leq c(2 k+n)^{n-1}(\sqrt{6(2 k+n)}+|z|)^{2 n},
$$

and so the assertion of the lemma is justified for $p>1$.

If $p=1$ then

$$
\int_{\mathbb{C}^{n}} f(u) \tau_{z} \check{\vartheta}_{k}^{n-1}(u) d u \leq\|f\|_{L^{1}(\omega)}\left\|\tau_{z} \check{\vartheta}_{k}^{n-1} \omega^{-1}\right\|_{\infty},
$$


and it remains to estimate $\left\|\tau_{z} \check{\vartheta}_{k}^{n-1} \omega^{-1}\right\|_{\infty}$. In view of the $A_{1}$ condition we may write

$$
\operatorname{essup}_{u \in B_{r}} \frac{1}{\omega(u)} \leq c \frac{\left|B_{r}\right|}{\omega\left(B_{r}\right)} \leq c \frac{r^{2 n}}{\omega\left(B_{1}\right)} \leq c r^{2 n}, \quad r \geq 1,
$$

and therefore

$$
\begin{aligned}
\operatorname{ess~sup}_{u \in \Gamma_{1}} \tau_{z} \check{\vartheta}_{k}^{n-1}(u) \omega(u)^{-1} & \leq(2 k+n)^{n-1} \operatorname{ess} \sup \omega(u)^{-1} \\
& \leq c(2 k+n)^{n-1}(\sqrt{6(2 k+n)}+|z|)^{2 n}, \\
\operatorname{ess~sup}_{u \in \Gamma_{2}^{m}} \tau_{z} \check{\vartheta}_{k}^{n-1}(u) \omega(u)^{-1} & \leq \exp \left(-\gamma 2^{2 m} 6(2 k+n)\right) \operatorname{ess\operatorname {sup}} \omega(u)^{-1} \\
u \in \Gamma_{2}^{m} & \leq c(\sqrt{6(2 k+n)}+|z|)^{2 n} \exp \left(-\gamma 2^{2 m}\right) 2^{2 n m}
\end{aligned}
$$

The conclusion follows.

REMARK 3.6. Let $f, p$ and $\omega$ be as in Lemma 3.5 and let $M \geq 0, m \geq 1$. Then, by the above proof and the estimate (2),

$$
\begin{aligned}
\left|f \times\left[\left(1+|\cdot|^{2}\right)^{M} \phi_{k}^{m-1}(\cdot)\right](z)\right| & \leq c|f| *\left[\left(1+|\cdot|^{2}\right)^{M} \vartheta_{k}^{m-1}(\cdot)\right](z) \\
& \leq c(2 k+m)^{m-1+M}(\sqrt{6(2 k+m)}+|z|)^{2 n}\|f\|_{L^{p}(\omega)}
\end{aligned}
$$

with $c$ independent of $k$. We will make use of this fact in what follows.

Lemma 3.7. Let $1 \leq p<\infty$ and $\omega \in A_{p}$. The subspace generated by $\left\{\Phi_{\alpha, \beta}: \alpha, \beta \in \mathbb{N}^{n}\right\}$ is dense in $L^{p}(\omega)$ and in $C_{0}\left(\mathbb{C}^{n}\right)$ with $\|\cdot\|_{\infty}$ norm.

Proof. According to Thangavelu [Th2, Theorem 1.4.4], the finite linear combinations of $\Phi_{\alpha, \beta}$ are dense in the Schwartz class $\mathcal{S}\left(\mathbb{C}^{n}\right)$. Thus the density in $C_{0}$ follows immediately. To prove the density in $L^{p}(\omega)$ we observe that by (4) and the dominated convergence theorem it follows that the convergence in $\mathcal{S}\left(\mathbb{C}^{n}\right)$ implies the convergence in $L^{p}(\omega)$, and hence functions from $\mathcal{S}\left(\mathbb{C}^{n}\right)$ may be approximated in $L^{p}(\omega)$ norm by finite linear combinations of $\Phi_{\alpha, \beta}$.

Corollary 3.8. Let $1 \leq p<\infty, \omega \in A_{p}$ and $f \in L^{p}(\omega)$. If $\left\langle f, \Phi_{\alpha, \beta}\right\rangle=0$ for all $\alpha, \beta \in \mathbb{N}^{n}$ then $f=0$.

Proof. If $p=1$ Lemma 3.7 gives $\langle f, g\rangle=0$ for each $g \in C_{0}\left(\mathbb{C}^{n}\right)$ and the conclusion follows. If $1<p<\infty$ then, again by Lemma 3.7, we get $\langle f, g\rangle=0$ for each $g \in L^{p^{\prime}}\left(\omega^{-p^{\prime} / p}\right)$ (recall that $\omega^{-p^{\prime} / p} \in A_{p^{\prime}}$ ). The claim is proved.

Let $1 \leq p<\infty$ and $\omega \in A_{p}$. Given $f \in L^{p}(\omega)$ we define its heat-diffusion integral by

$$
g(t, z)=\sum_{k=0}^{\infty} e^{-t(2 k+n)} Q_{k} f(z), \quad t>0 .
$$


Note that the above series converges pointwise by Lemma 3.5 and in $L^{p}(\omega)$ by Lemma 3.4. We may express $g(t, z)$ as a twisted convolution with a kernel $G_{t}$ by writing

$$
\begin{aligned}
g(t, z) & =\sum_{k=0}^{\infty} e^{-t(2 k+n)} f \times \phi_{k}^{n-1}(z) \\
& =\sum_{k=0}^{\infty} e^{-t(2 k+n)} \int_{\mathbb{C}^{n}} f(z-u) \phi_{k}^{n-1}(u) e^{i \operatorname{Im}\langle z, u\rangle / 2} d u \\
& =\int_{\mathbb{C}^{n}} f(z-u)\left(\sum_{k=0}^{\infty} e^{-t(2 k+n)} \phi_{k}^{n-1}(u)\right) e^{i \operatorname{Im}\langle z, u\rangle / 2} d u \\
& =f \times G_{t}(z) .
\end{aligned}
$$

Interchanging the order of summation and integration is justified by Remark 3.6 since

$$
\begin{aligned}
& \sum_{k=0}^{\infty} e^{-t(2 k+n)} \int_{\mathbb{C}^{n}}\left|f(z-u) \phi_{k}^{n-1}(u)\right| d u \leq c \sum_{k=0}^{\infty} e^{-t(2 k+n)}|f| * \vartheta_{k}^{n-1}(z) \\
& \quad \leq c\|f\|_{L^{p}(\omega)} \sum_{k=0}^{\infty} e^{-t(2 k+n)}(2 k+n)^{n-1}(\sqrt{6(2 k+n)}+|z|)^{2 n}<\infty .
\end{aligned}
$$

Using the generating formula $[\mathrm{L},(4.17 .3)]$ we get

$$
G_{t}(z)=\frac{1}{(4 \pi \sinh t)^{n}} \exp \left(-\frac{1}{4}|z|^{2} \operatorname{coth} t\right), \quad t>0 .
$$

Proposition 3.9. Let $1 \leq p<\infty, \omega \in A_{p}$ and $f \in L^{p}(\omega)$. The heatdiffusion integral $g(t, z)$ of $f$ is a smooth function on $\mathbb{R}_{+} \times \mathbb{R}^{2 n}$. Moreover, it satisfies

$$
\left(\partial / \partial t+\widetilde{\Delta}_{z}\right) g(t, z)=0 .
$$

Proof. Let $E$ be a compact subset of $\mathbb{C}^{n}$. Then $\sup _{z \in E}\left|Q_{k} f(z)\right|$ grows polynomially in $k$ by Lemma 3.5. Therefore we may differentiate term by term with respect to $t$ the series defining $g(t, z)$. We obtain

$$
\frac{\partial^{m}}{\partial t^{m}} g(t, z)=\sum_{k=0}^{\infty}(-1)^{m}(2 k+n)^{m} e^{-t(2 k+n)} Q_{k} f(z),
$$

RHS being continuous in $(t, z)$ since each $Q_{k}(z)$ is a continuous function of $z$ and the series is convergent almost uniformly in $(t, z)$.

For $z \in \mathbb{C}^{n}$ we write $z=x+i y, x, y \in \mathbb{R}^{n}$. To prove the smoothness of (6) in $z$ we first focus on

$$
\partial_{x}^{\alpha} \partial_{y}^{\beta} Q_{k} f(z)=\partial_{x}^{\alpha} \partial_{y}^{\beta} \int_{\mathbb{C}^{n}} f(u) \phi_{k}^{n-1}(z-u) e^{i \operatorname{Im}\langle z, z-u\rangle / 2} d u .
$$


We claim that the differentiation may be taken under the integral sign. Define

$$
\begin{aligned}
\Upsilon_{k}^{n-1}(z, u) & =\phi_{k}^{n-1}(z-u) e^{i \operatorname{Im}\langle z, z-u\rangle / 2} \\
& =\frac{1}{(2 \pi)^{n}} L_{k}^{n-1}\left(\frac{|z-u|^{2}}{2}\right) e^{-|z-u|^{2} / 4+i \operatorname{Im}\langle z, z-u\rangle / 2} .
\end{aligned}
$$

Observe that

$$
\begin{aligned}
& \partial_{x}^{\alpha} \partial_{y}^{\beta} \Upsilon_{k}^{n-1}(z, u) \\
& \quad=e^{-|z-u|^{2} / 4+i \operatorname{Im}\langle z, z-u\rangle / 2} \sum_{|\nu| \leq|\alpha|+|\beta|} \partial^{|\nu|} L_{k}^{n-1}\left(\frac{|z-u|^{2}}{2}\right) P_{\nu}(z, z-u),
\end{aligned}
$$

where $P_{\nu}$ are polynomials on $\mathbb{C}^{n} \times \mathbb{C}^{n}$.

Fix $z \in \mathbb{C}^{n}$ and set $e_{1}=(1,0, \ldots, 0) \in \mathbb{R}^{n}$. For $-1<\varepsilon<1$, by the mean value theorem we have

$$
\varepsilon^{-1}\left|\Upsilon_{k}^{n-1}\left(z+\varepsilon e_{1}, u\right)-\Upsilon_{k}^{n-1}(z, u)\right|=\left|\partial_{x}^{e_{1}} \Upsilon_{k}^{n-1}\left(z+\theta e_{1}, u\right)\right|
$$

for some $\theta \in(-1,1)$. In view of $(7)$ the function

$$
\Upsilon^{*}(z, \cdot)=\sup _{|\theta|<1}\left|\partial_{x}^{e_{1}} \Upsilon_{k}^{n-1}\left(z+\theta e_{1}, \cdot\right)\right|
$$

is bounded and rapidly decreasing. Moreover, (4) implies $\Upsilon^{*}(z, \cdot) \in$ $L^{p^{\prime}}\left(\omega^{-p^{\prime} / p}\right)$ and therefore integrability of $\Upsilon^{*}(z, u)|f(u)|$ is justified by Hölder's inequality. Hence the dominated convergence theorem may be applied and we obtain

$$
\partial_{x}^{e_{1}} Q_{k} f(z)=\int_{\mathbb{C}^{n}} f(u) \partial_{x}^{e_{1}} \Upsilon_{k}^{n-1}(z, u) d u
$$

Our claim follows by repeating the above arguments for the remaining partial derivatives.

Now our aim is to show that the series

$$
\sum_{k=0}^{\infty}(-1)^{m}(2 k+n)^{m} e^{-t(2 k+n)} \partial_{x}^{\alpha} \partial_{y}^{\beta} Q_{k} f(z)
$$

is almost uniformly convergent. Since each term is a continuous function this will finish the proof of smoothness of $g(t, z)$ (the continuity in $z$ of each term is checked by the mean value theorem, (7) and the dominated convergence theorem).

We have $([\mathrm{L},(4.18 .6)]) \partial^{m} L_{k}^{n-1}=(-1)^{m} L_{k-m}^{n+m-1}$ (we use the convention that $L_{k-m}^{n+m-1}=0$ if $\left.k<m\right)$. Therefore, by (7),

$$
\left|\partial_{x}^{\alpha} \partial_{y}^{\beta} \Upsilon_{k}^{n-1}(z, u)\right| \leq c\left(1+|z|^{2}\right)^{M}\left(1+|z-u|^{2}\right)^{M} \sum_{m=0}^{|\alpha|+|\beta|}\left|\phi_{k-m}^{n+m-1}(z-u)\right|
$$


with $c$ independent of $k \in \mathbb{N}$ and the convention that $\phi_{k-m}^{n+m-1}=0$ for $k<m$. This, in view of Remark 3.6, gives

$$
\begin{aligned}
& \left|\partial_{x}^{\alpha} \partial_{y}^{\beta} Q_{k} f(z)\right| \\
& \quad \leq c\|f\|_{L^{p}(\omega)}\left(1+|z|^{2}\right)^{M}(2 k+n)^{n-1+|\alpha|+|\beta|+M}(\sqrt{6(2 k+n)}+|z|)^{2 n} .
\end{aligned}
$$

Hence $\sup _{z \in E}\left|\partial_{x}^{\alpha} \partial_{y}^{\beta} Q_{k} f(z)\right|$ grows polynomially in $k$ and the almost uniform convergence of (8) is justified.

To verify the heat equation (5) we differentiate term by term the series defining $g(t, z)$ and use the fact that $L_{k}^{n-1}$ satisfies ([L, (4.18.7)])

$$
x \partial^{2} L_{k}^{n-1}(x)+(n-x) \partial L_{k}^{n-1}(x)+k L_{k}^{n-1}(x)=0 .
$$

The computation makes no difficulties and is omitted.

Theorem 3.10. Let $1 \leq p<\infty, \omega \in A_{p}$ and $f \in L^{p}(\omega)$. Let $g(t, z)$ be the heat-diffusion integral of $f$. Then

(a) $\sup _{t>0}|g(t, z)| \leq M f(z), z \in \mathbb{C}^{n}$;

(b) $\|g(t, \cdot)\|_{L^{p}(\omega)} \leq C(\cosh t)^{-n}\|f\|_{L^{p}(\omega)}$;

(c) $\|g(t, \cdot)-f\|_{L^{p}(\omega)} \rightarrow 0, t \rightarrow 0^{+}$;

(d) $g(t, z) \rightarrow f(z)$ a.e., $t \rightarrow 0^{+}$.

Moreover, the family $\left\{T_{t}\right\}_{t>0}, T_{t} f(z)=g(t, z)$, is a strongly continuous and uniformly bounded semigroup of operators on $L^{p}(\omega)$.

Proof. Observe that

$$
G_{t}(z)=(\cosh t)^{-n} W_{\sqrt{\tanh t}}(z)
$$

where $W(x)=(4 \pi)^{-n} \exp \left(-|x|^{2} / 4\right)$ is the Gauss-Weierstrass kernel in $\mathbb{R}^{2 n} \simeq \mathbb{C}^{n}$ and $W_{\varepsilon}(\cdot)=\varepsilon^{-2 n} W(\cdot / \varepsilon)$. Since $|g(t, z)| \leq|f| * G_{t}(z)$ assertions (a) and (b) follow from Lemma 3.2 and Remark 3.3. Items (c) and (d) are justified by standard arguments with the aid of (a), (b) and Lemma 3.7.

The semigroup property of $\left\{T_{t}\right\}_{t>0}$ is easily verified for $f \in \operatorname{lin}\left\{\Phi_{\alpha, \beta}\right\}$; hence it holds for any $f \in L^{p}(\omega)$ in view of Lemma 3.7 and (b). Strong continuity follows by standard arguments, similarly to (c).

We now pass to Poisson integrals. Let $1 \leq p<\infty$ and $\omega \in A_{p}$. Given $f \in L^{p}(\omega)$ we define its Poisson integral by

$$
f(t, z)=\sum_{k=0}^{\infty} e^{-t \sqrt{2 k+n}} Q_{k} f(z), \quad t>0 .
$$

The above series converges pointwise by Lemma 3.5 and in $L^{p}(\omega)$ by Lemma 3.4. Using the well known formula

$$
e^{-\beta t}=\frac{t}{\sqrt{4 \pi}} \int_{0}^{\infty} e^{-\beta^{2} s} s^{-3 / 2} e^{-t^{2} /(4 s)} d s, \quad t>0, \beta \geq 0,
$$


we express $f(t, z)$ as the twisted convolution with a kernel $P_{t}$ :

$$
\begin{aligned}
f(t, z)= & \sum_{k=0}^{\infty} e^{-t \sqrt{2 k+n}} f \times \phi_{k}^{n-1}(z) \\
= & \sum_{k=0}^{\infty} \frac{t}{\sqrt{4 \pi}} \int_{0}^{\infty} e^{-s(2 k+n)} s^{-3 / 2} e^{-t^{2} /(4 s)} d s \\
& \cdot \int_{\mathbb{C}^{n}} f(z-u) \phi_{k}^{n-1}(u) e^{i \operatorname{Im}\langle z, u\rangle / 2} d u \\
= & \int_{\mathbb{C}^{n}} \frac{t}{\sqrt{4 \pi}} \int_{0}^{\infty}\left(s^{-3 / 2} e^{-t^{2} /(4 s)} \sum_{k=0}^{\infty} e^{-s(2 k+n)} \phi_{k}^{n-1}(u)\right) d s \\
& \cdot f(z-u) e^{i \operatorname{Im}\langle z, u\rangle / 2} d u \\
= & f \times P_{t}(z),
\end{aligned}
$$

where

$$
P_{t}(z)=\frac{t}{\sqrt{4 \pi}} \int_{0}^{\infty} G_{s}(z) s^{-3 / 2} e^{-t^{2} /(4 s)} d s .
$$

Interchanging the order of summation and integration is justified by Fubini's theorem since, by Remark 3.6,

$$
\begin{aligned}
& \sum_{k=0}^{\infty} \frac{t}{\sqrt{4 \pi}} \int_{0}^{\infty} e^{-s(2 k+n)} s^{-3 / 2} e^{-t^{2} /(4 s)} \int_{\mathbb{C}^{n}}\left|f(z-u) \phi_{k}^{n-1}(u)\right| d u d s \\
& \quad \leq c \sum_{k=0}^{\infty}|f| * \vartheta_{k}^{n-1}(z) \frac{t}{\sqrt{4 \pi}} \int_{0}^{\infty} e^{-s(2 k+n)} s^{-3 / 2} e^{-t^{2} /(4 s)} d s \\
& \quad \leq c\|f\|_{L^{p}(\omega)} \sum_{k=0}^{\infty} e^{-t \sqrt{2 k+n}}(2 k+n)^{n-1}(\sqrt{6(2 k+n)}+|z|)^{2 n}<\infty .
\end{aligned}
$$

Note that by (11) we obtain the following subordination formula:

$$
f(t, z)=\frac{t}{\sqrt{4 \pi}} \int_{0}^{\infty} g(s, z) s^{-3 / 2} e^{-t^{2} /(4 s)} d s, \quad t>0 .
$$

Proposition 3.11. Let $1 \leq p<\infty, \omega \in A_{p}$ and $f \in L^{p}(\omega)$. Then the Poisson integral $f(t, z)$ of $f$ is a smooth function on $\mathbb{R}_{+} \times \mathbb{R}^{2 n}$. Moreover, it satisfies

$$
\left(\partial^{2} / \partial t^{2}-\widetilde{\Delta}_{z}\right) f(t, z)=0 .
$$

Proof. The proof is very similar to that of Proposition 3.9. We omit the details.

Theorem 3.12. Assume that $1 \leq p<\infty, \omega \in A_{p}$ and $f \in L^{p}(\omega)$. Let $f(t, z)$ be the Poisson integral of $f$. Then 
(a) $\sup _{t>0}|f(t, z)| \leq M f(z), z \in \mathbb{C}^{n}$;

(b) $\|f(t, \cdot)\|_{L^{p}(\omega)} \leq C e^{-t \sqrt{n}}\|f\|_{L^{p}(\omega)}$;

(c) $\|f(t, \cdot)-f\|_{L^{p}(\omega)} \rightarrow 0, t \rightarrow 0^{+}$;

(d) $f(t, z) \rightarrow f(z)$ a.e., $t \rightarrow 0^{+}$.

Moreover, the family $\left\{P_{t}\right\}_{t>0}, P_{t} f(z)=f(t, z)$, is a strongly continuous and uniformly bounded semigroup of operators on $L^{p}(\omega)$.

Proof. Using the subordination formula (12) and (a) of Theorem 3.10 we get

$$
|f(t, z)| \leq \int_{0}^{\infty} M f(z) \frac{t}{\sqrt{4 \pi}} s^{-3 / 2} e^{-t^{2} /(4 s)} d s
$$

and so (a) follows. To prove (b) we apply Minkowski's integral inequality and Theorem 3.10(b) to obtain

$$
\begin{aligned}
\|f(t, \cdot)\|_{L^{p}(\omega)} & \leq \int_{0}^{\infty}\|g(s, \cdot)\|_{L^{p}(\omega)} \frac{t}{\sqrt{4 \pi}} s^{-3 / 2} e^{-t^{2} /(4 s)} d s \\
& \leq c\|f\|_{L^{p}(\omega)} \int_{0}^{\infty}(\cosh s)^{-n} \frac{t}{\sqrt{4 \pi}} s^{-3 / 2} e^{-t^{2} /(4 s)} d s \\
& \leq c e^{-t \sqrt{n}}\|f\|_{L^{p}(\omega)} .
\end{aligned}
$$

The rest of the proof is analogous to the proof of Theorem 3.10.

REMARK 3.13. Theorem 3.10 shows that the condition $\omega \in A_{p}$ is sufficient to have $\|g(t, z)\|_{L^{p}(\omega)} \leq C\|f\|_{L^{p}(\omega)}$. However, it is not necessary. For $1<p<\infty$ it was proved in [KeTh] that a certain local $A_{p}$ condition is both necessary and sufficient for the above weighted norm inequality to hold. Some results of this type were also obtained in the case of the one-dimensional system of Laguerre functions defined in Remark 6.8 below.

REMARK 3.14. Most of the results of this section are valid for the space $L^{\infty}\left(\mathbb{C}^{n}\right)$. More precisely, Proposition 3.1, Lemma 3.4 (and so Lemma 3.5), Proposition 3.9 and Proposition 3.11 remain valid if we replace $L^{p}(\omega)$ by $L^{\infty}$. Moreover, Theorem 3.10 and Theorem 3.12, except (c) and (d), also remain valid with $L^{\infty}$ replacing $L^{p}(\omega)$. Concerning (c) and (d), we have $\|g(t, \cdot)-f\|_{\infty} \rightarrow 0$ and $\|f(t, \cdot)-f\|_{\infty} \rightarrow 0, t \rightarrow 0^{+}$, but only for $f \in C_{0}\left(\mathbb{C}^{n}\right)$.

Remark 3.15. If $1 \leq p \leq \infty$ and $f \in L^{p}\left(\mathbb{C}^{n}\right)$ (the case $\omega \equiv 1$ ) then Theorem 3.10(b) and Theorem 3.12(b) hold with $C=1$. In particular this means that $\left\{T_{t}\right\}$ and $\left\{P_{t}\right\}$ are semigroups of contractions on $L^{p}\left(\mathbb{C}^{n}\right), 1 \leq p \leq \infty$.

4. Laguerre expansions; system $\left\{\ell_{k}^{\alpha}\right\}$. Let $k=\left(k_{1}, \ldots, k_{d}\right) \in \mathbb{N}^{d}$ and $\alpha=\left(\alpha_{1}, \ldots, \alpha_{d}\right) \in(-1, \infty)^{d}$ be multi-indices. The Laguerre function $\ell_{k}^{\alpha}$ on 
$\mathbb{R}_{+}^{d}$ is defined as

$$
\ell_{k}^{\alpha}(x)=\ell_{k_{1}}^{\alpha_{1}}\left(x_{1}\right) \cdot \ldots \cdot \ell_{k_{d}}^{\alpha_{d}}\left(x_{d}\right), \quad x=\left(x_{1}, \ldots, x_{d}\right) \in \mathbb{R}_{+}^{d},
$$

where $\ell_{k_{i}}^{\alpha_{i}}$ are the one-dimensional Laguerre functions given by

$$
\ell_{k_{i}}^{\alpha_{i}}\left(x_{i}\right)=\left(\frac{\Gamma\left(k_{i}+1\right)}{\Gamma\left(k_{i}+\alpha_{i}+1\right)}\right)^{1 / 2} L_{k_{i}}^{\alpha_{i}}\left(x_{i}\right) e^{-x_{i} / 2}, \quad x_{i}>0, i=1, \ldots, d .
$$

Each $\ell_{k}^{\alpha}$ is an eigenfunction of the differential operator

$$
L=\sum_{i=1}^{d}\left(x_{i} \frac{\partial^{2}}{\partial x_{i}^{2}}+\left(\alpha_{i}+1\right) \frac{\partial}{\partial x_{i}}-\frac{x_{i}}{4}\right)
$$

the corresponding eigenvalue being $-|k|-(|\alpha|+d) / 2$. The operator $-L$ is positive and symmetric in $L^{2}\left(\mathbb{R}_{+}^{d}, x^{\alpha} d x\right)$. Moreover, the system $\left\{\ell_{k}^{\alpha}: k \in \mathbb{N}^{d}\right\}$ is an orthonormal basis in $L^{2}\left(\mathbb{R}_{+}^{d}, x^{\alpha} d x\right)$.

The following estimate of $\ell_{k}^{\alpha}$ is crucial for further considerations:

$$
\left|\ell_{k}^{\alpha}(x)\right| \leq c \prod_{i=1}^{d} \Phi_{k_{i}}^{\alpha_{i}}\left(x_{i}\right), \quad x \in \mathbb{R}_{+}^{d}
$$

where

$$
\Phi_{k_{i}}^{\alpha_{i}}\left(x_{i}\right)= \begin{cases}\left(2 k_{i}+\left|\alpha_{i}\right|+1\right)^{\left|\alpha_{i}\right| / 2}, & 0<x_{i} \leq 3\left(2 k_{i}+\left|\alpha_{i}\right|+1\right), \\ \exp \left(-\gamma x_{i}\right), & x_{i}>3\left(2 k_{i}+\left|\alpha_{i}\right|+1\right) .\end{cases}
$$

Here $c$ and $\gamma$ are independent of $k$ and $x$. The estimate (13) is a consequence of Muckenhoupt's generalization [Mu2] of the classical estimates due to Askey and Wainger [AW].

Let $1 \leq p<\infty$. We denote by $A_{p}^{\alpha}=A_{p}\left(\mathbb{R}_{+}^{d}, d \mu_{\alpha}\right)$ the class of $A_{p}$ weights on $\mathbb{R}_{+}^{d}$ with respect to the (doubling) measure $\mu_{\alpha}(d x)=x^{\alpha} d x$. More precisely, $A_{p}^{\alpha}$ is the class of all nonnegative functions $\omega \in L_{\text {loc }}^{1}\left(\mathbb{R}_{+}^{d}, d \mu_{\alpha}\right)$ such that $\omega^{-p^{\prime} / p} \in L_{\mathrm{loc}}^{1}\left(\mathbb{R}_{+}^{d}, d \mu_{\alpha}\right)$ and

$$
\sup _{Q \in \mathcal{B}}\left[\frac{1}{\mu_{\alpha}(Q)} \int_{Q} \omega(x) \mu_{\alpha}(d x)\right]\left[\frac{1}{\mu_{\alpha}(Q)} \int_{Q} \omega(x)^{-p^{\prime} / p} \mu_{\alpha}(d x)\right]^{p / p^{\prime}}<\infty
$$

if $1<p<\infty$, or

$$
\sup _{Q \in \mathcal{B}} \frac{1}{\mu_{\alpha}(Q)} \int_{Q} \omega(x) \mu_{\alpha}(d x) \operatorname{ess} \sup _{x \in Q} \frac{1}{\omega(x)}<\infty
$$

if $p=1$. Here $\mathcal{B}$ denotes the class of all sets of the form $Q=\widetilde{Q} \cap \mathbb{R}_{+}^{d}$, where $\widetilde{Q}$ is a cube (with sides parallel to the coordinate axes) in $\mathbb{R}^{d}$ with center in $\mathbb{R}_{+}^{d}$. 
For $r>0$ denote by $Q_{r}$ the cube $(0, r)^{d}$. Given $1 \leq p<\infty$ and $\omega \in A_{p}^{\alpha}$ we have

$$
\omega\left(Q_{r}\right)=\int_{Q_{r}} \omega(x) \mu_{\alpha}(d x) \leq c r^{(d+|\alpha|) p}, \quad r \geq 1 .
$$

Indeed, if $1<p<\infty$ and $r \geq 1$ then, by Hölder's inequality and the $A_{p}^{\alpha}$ condition,

$$
\mu_{\alpha}\left(Q_{1}\right) \leq c \omega\left(Q_{1}\right)^{1 / p} \mu_{\alpha}\left(Q_{r}\right) \omega\left(Q_{r}\right)^{-1 / p} .
$$

If $p=1$ then the $A_{1}^{\alpha}$ condition (15) gives

$$
\mu_{\alpha}\left(Q_{1}\right) \leq \int_{Q_{1}} \omega(x) \mu_{\alpha}(d x) \operatorname{esssup}_{x \in Q_{r}} \frac{1}{\omega(x)} \leq c \omega\left(Q_{1}\right) \mu_{\alpha}\left(Q_{r}\right) \omega\left(Q_{r}\right)^{-1} .
$$

Since $\mu_{\alpha}\left(Q_{r}\right)=c r^{d+|\alpha|}$ the inequality in (16) follows.

Lemma 4.1. Let $1 \leq p<\infty$ and $\omega \in A_{p}^{\alpha}$. There exist constants $\delta=\delta(\alpha)$ and $c$ independent of $k \in \mathbb{N}^{d}$ such that

$$
\left\|\ell_{k}^{\alpha}\right\|_{L^{p}\left(\omega d \mu_{\alpha}\right)} \leq c(2|k|+\|\alpha\|+1)^{\delta d},
$$

where $\|\alpha\|=\sum_{i=1}^{d}\left|\alpha_{i}\right|$.

Proof. Set $\lambda_{i}=3\left(2 k_{i}+\left|\alpha_{i}\right|+1\right), i=1, \ldots, d$, and $\lambda^{*}=\max \left\{\lambda_{i}:\right.$ $1 \leq i \leq d\}$. For $S \subset\{1, \ldots, d\}$ we define

$$
\Gamma_{k}^{\alpha}(S)=\left\{x \in \mathbb{R}_{+}^{d}: x_{j}>\lambda_{j} \text { for } j \in S \& x_{j} \leq \lambda_{j} \text { for } j \notin S\right\} .
$$

Note that $\left\{\Gamma_{k}^{\alpha}(S): S \subset\{1, \ldots, d\}\right\}$ is a decomposition of $\mathbb{R}_{+}^{d}$ into $2^{d}$ disjoint subsets. Therefore, to finish the proof it is sufficient to obtain a proper estimate of

$$
I_{k}^{\alpha}(S)=\int_{\Gamma_{k}^{\alpha}(S)}\left|\ell_{k}^{\alpha}(x)\right|^{p} \omega(x) \mu_{\alpha}(d x)
$$

If $S=\emptyset$ then by (13) and (16) we have

$$
\begin{aligned}
I_{k}^{\alpha}(S) & \leq c \int_{\Gamma_{k}^{\alpha}(S)}\left(\max _{1 \leq i \leq d} \lambda_{i}{ }^{\left|\alpha_{i}\right| / 2}\right)^{d p} \omega(x) \mu_{\alpha}(d x) \\
& \leq c(2|k|+\|\alpha\|+1)^{\|\alpha\| d p / 2} \int_{Q_{\lambda^{*}}} \omega(x) \mu_{\alpha}(d x) \\
& \leq c(2|k|+\|\alpha\|+1)^{\|\alpha\| d p / 2}\left(\lambda^{*}\right)^{(d+|\alpha|) p} \leq c(2|k|+\|\alpha\|+1)^{(1+3\|\alpha\| / 2) d p} .
\end{aligned}
$$

If $S \neq \emptyset$ we divide $\Gamma_{k}^{\alpha}(S)$ into disjoint subsets $\Gamma_{k}^{\alpha}(S, m), m \in \mathbb{N}$, defined as follows:

$$
\Gamma_{k}^{\alpha}(S, 0)=\widetilde{\Gamma}_{k}^{\alpha}(S, 0), \quad \Gamma_{k}^{\alpha}(S, m)=\widetilde{\Gamma}_{k}^{\alpha}(S, m) \backslash \widetilde{\Gamma}_{k}^{\alpha}(S, m-1), \quad m \geq 1,
$$

where

$$
\widetilde{\Gamma}_{k}^{\alpha}(S, m)=\left\{x \in \mathbb{R}_{+}^{d}: \lambda_{j}<x_{j} \leq 2^{m+1} \lambda_{j} \text { for } j \in S \& x_{j} \leq \lambda_{j} \text { for } j \notin S\right\} .
$$


Now, using (13) and (16) we obtain

$$
\begin{aligned}
I_{k}^{\alpha}(S) & \leq c \sum_{m=0}^{\infty} \int_{\Gamma_{k}^{\alpha}(S, m)}\left[\exp \left(-\gamma \sum_{j \in S} x_{j}\right) \prod_{j \notin S}\left(\lambda_{j}\right)^{\left|\alpha_{j}\right| / 2}\right]^{p} \omega(x) \mu_{\alpha}(d x) \\
& \leq c \prod_{j=1}^{d}\left(\lambda_{j}\right)^{|\alpha| p / 2} \sum_{m=0}^{\infty} \exp \left(-\gamma p 2^{m} \min _{j \in S} \lambda_{j}\right) \int_{\Gamma_{k}^{\alpha}(S, m)} \omega(x) \mu_{\alpha}(d x) \\
& \leq c(2|k|+\|\alpha\|+1)^{\|\alpha\| d p / 2} \sum_{m=0}^{\infty} \exp \left(-\gamma p 2^{m}\right) \int_{Q_{2^{m+1} \lambda^{*}}} \omega(x) \mu_{\alpha}(d x) \\
& \leq c(2|k|+\|\alpha\|+1)^{\|\alpha\| d p / 2} \sum_{m=0}^{\infty} \exp \left(-\gamma p 2^{m}\right)\left(2^{m+1} \lambda^{*}\right)^{(d+|\alpha|) p} \\
& \leq c(2|k|+\|\alpha\|+1)^{(1+3\|\alpha\| / 2) d p} .
\end{aligned}
$$

The conclusion follows.

Lemma 4.2. Let $1 \leq p<\infty$ and $\omega \in A_{p}^{\alpha}$. The Fourier-Laguerre coefficients $\left\langle\ell_{k}^{\alpha}, f\right\rangle=\int_{\mathbb{R}_{+}^{d}} \ell_{k}^{\alpha}(x) f(x) \mu_{\alpha}(d x)$ exist for $f \in L^{p}\left(\omega d \mu_{\alpha}\right)$. Moreover, there exist constants $\delta=\delta(\alpha)$ and $c$ independent of $k \in \mathbb{N}^{d}$ such that

$$
\left|\left\langle\ell_{k}^{\alpha}, f\right\rangle\right| \leq c(2|k|+\|\alpha\|+1)^{\delta d}\|f\|_{L^{p}\left(\omega d \mu_{\alpha}\right)} .
$$

Proof. For $1<p<\infty$ Hölder's inequality implies

$$
\left|\left\langle\ell_{k}^{\alpha}, f\right\rangle\right| \leq\left[\int_{\mathbb{R}_{+}^{d}}\left|\ell_{k}^{\alpha}(x)\right|^{p^{\prime}} \omega(x)^{-p^{\prime} / p} \mu_{\alpha}(d x)\right]^{1 / p^{\prime}}\|f\|_{L^{p}\left(\omega d \mu_{\alpha}\right)},
$$

and since $\omega^{-p^{\prime} / p} \in A_{p^{\prime}}^{\alpha}$, Lemma 4.1 gives (17).

The case $p=1$ is less straightforward. We use the notation from the proof of Lemma 4.1. We have

$$
\begin{aligned}
\left|\left\langle\ell_{k}^{\alpha}, f\right\rangle\right| & \leq \sum_{S \subset\{1, \ldots, d\}} \operatorname{essiup}_{y \in \Gamma_{k}^{\alpha}(S)} \frac{1}{\omega(y)}\left|\ell_{k}^{\alpha}(y)\right| \int_{\Gamma_{k}^{\alpha}(S)}|f(x)| \omega(x) \mu_{\alpha}(d x) \\
& \leq\|f\|_{L^{1}\left(\omega d \mu_{\alpha}\right)} \max _{S \subset\{1, \ldots, d\}} \operatorname{ess~sup}_{y \in \Gamma_{k}^{\alpha}(S)} \frac{1}{\omega(y)}\left|\ell_{k}^{\alpha}(y)\right| .
\end{aligned}
$$

If $S=\emptyset$ then by the $A_{1}^{\alpha}$ condition, (13) and (16) we obtain

$$
\begin{aligned}
\operatorname{ess}_{y \in \Gamma_{k}^{\alpha}(S)} \frac{1}{\omega(y)}\left|\ell_{k}^{\alpha}(y)\right| & \leq c(2|k|+\|\alpha\|+1)^{\|\alpha\| d / 2} \underset{y \in Q_{\lambda^{*}}}{\operatorname{ess} \sup \frac{1}{\omega(y)}} \\
& \leq c(2|k|+\|\alpha\|+1)^{(1+3\|\alpha\| / 2) d} .
\end{aligned}
$$


If $S \neq \emptyset$ we use again the $A_{1}^{\alpha}$ condition, (13) and (16) to get

$$
\begin{aligned}
\operatorname{ess~sup}_{y \in \Gamma_{k}^{\alpha}(S)} \frac{1}{\omega(y)}\left|\ell_{k}^{\alpha}(y)\right| & \leq \sup _{m \in \mathbb{N}} \operatorname{ess}_{y \in \Gamma_{k}^{\alpha}(S, m)} \frac{1}{\omega(y)}\left|\ell_{k}^{\alpha}(y)\right| \\
& \leq c(2 k+\|\alpha\|+1)^{\|\alpha\| d / 2} \sup _{m \in \mathbb{N}} e^{-\gamma 2^{m}} \operatorname{ess~sup~}_{y \in Q_{2^{m+1} \lambda^{*}}} \frac{1}{\omega(y)} \\
& \leq c(2 k+\|\alpha\|+1)^{(1+3\|\alpha\| / 2) d .}
\end{aligned}
$$

The proof is finished.

Lemma 4.3. Let $1 \leq p<\infty$ and $\omega \in A_{p}^{\alpha}$. The subspace spanned by $\left\{\ell_{k}^{\alpha}: k \in \mathbb{N}^{d}\right\}$ is dense in $L^{p}\left(\omega d \mu_{\alpha}\right)$ and in $C_{0}\left(\mathbb{R}_{+}^{d}\right)$ with $\|\cdot\|_{\infty}$ norm.

Proof. It is sufficient to approximate functions from $C_{\mathrm{c}}^{\infty}\left(\mathbb{R}_{+}^{d}\right)$ by linear combinations of $\ell_{k}^{\alpha}$. We first consider the case of $L^{p}\left(\omega d \mu_{\alpha}\right)$.

Fix $f \in C_{\mathrm{c}}^{\infty}\left(\mathbb{R}_{+}^{d}\right)$ and define

$$
S_{N} f=\sum_{|k| \leq N}\left\langle\ell_{k}^{\alpha}, f\right\rangle \ell_{k}^{\alpha}
$$

We will show that there exists a subsequence of $\left\{S_{N} f\right\}$ convergent to $f$ in $L^{p}\left(\omega d \mu_{\alpha}\right)$. Since $S_{N} f \rightarrow f$ in $L^{2}\left(d \mu_{\alpha}\right)$, there exists a subsequence $S_{N_{k}} f$ convergent to $f \mu_{\alpha}$-a.e. and thus $S_{N_{k}} f \rightarrow f$ a.e. Next, observe that by the symmetry of $-L$ we have, for $m \in \mathbb{N}$,

$$
\left\langle\ell_{k}^{\alpha}, f\right\rangle=\left\langle(-L)^{-m} \ell_{k}^{\alpha},(-L)^{m} f\right\rangle=\left(|k|+\frac{|\alpha|+d}{2}\right)^{-m}\left\langle\ell_{k}^{\alpha},(-L)^{m} f\right\rangle,
$$

hence, by the Schwarz inequality,

$$
\left|S_{N} f(x)\right| \leq \sum_{k \in \mathbb{N}^{d}}\left\|(-L)^{m} f\right\|_{L^{2}\left(d \mu_{\alpha}\right)}\left(|k|+\frac{|\alpha|+d}{2}\right)^{-m}\left|\ell_{k}^{\alpha}(x)\right| .
$$

Therefore, for $1<p<\infty$, by Hölder's inequality we get

$$
\begin{aligned}
\left|S_{N} f(x)\right|^{p} \leq & c\left(\sum_{k \in \mathbb{N}^{d}}\left(|k|+\frac{|\alpha|+d}{2}\right)^{-m}\right)^{p / p^{\prime}} \\
& \times \sum_{k \in \mathbb{N}^{d}}\left(|k|+\frac{|\alpha|+d}{2}\right)^{-m}\left|\ell_{k}^{\alpha}(x)\right|^{p} .
\end{aligned}
$$

Now, Lemma 4.1 implies, for $m$ sufficiently large,

$$
\int_{\mathbb{R}_{+}^{d}}\left|S_{N} f(x)\right|^{p} \omega(x) \mu_{\alpha}(d x) \leq c \sum_{k \in \mathbb{N}^{d}}\left(|k|+\frac{|\alpha|+d}{2}\right)^{-m}(2|k|+\|\alpha\|+1)^{\delta d p}<\infty .
$$

To show that $\left\|S_{N_{k}} f-f\right\|_{L^{p}\left(\omega d \mu_{\alpha}\right)} \rightarrow 0$ as $k \rightarrow \infty$, we apply the dominated convergence theorem (the majorant is $\left(\Upsilon+|f|^{p}\right) \omega$, where $\Upsilon$ is the RHS in (19) if $p=1$ or in (20) if $p>1$ ). 
We pass to the case of $C_{0}\left(\mathbb{R}_{+}^{d}\right)$. Since $S_{N_{k}} f \rightarrow f$ a.e., the proof is finished once we show that $S_{N} f$ is uniformly fundamental.

Let $1 \leq N<M$. By (13), (18) and the Schwarz inequality we obtain

$$
\begin{aligned}
& \left|S_{M} f(x)-S_{N} f(x)\right| \leq \sum_{n=N+1}^{M} \sum_{|k|=n}\left|\left\langle\ell_{k}^{\alpha}, f\right\rangle\right|\left|\ell_{k}^{\alpha}(x)\right| \\
& \leq c \sum_{n=N+1}^{M} \sum_{|k|=n}\left\|(-L)^{m} f\right\|_{L^{2}\left(d \mu_{\alpha}\right)}\left(|k|+\frac{|\alpha|+d}{2}\right)^{-m}(2|k|+\|\alpha\|+1)^{\|\alpha\| d / 2} .
\end{aligned}
$$

The last expression tends to 0 as $N, M \rightarrow \infty$, if only $m$ is chosen sufficiently large.

Corollary 4.4. Let $1 \leq p<\infty, \omega \in A_{p}^{\alpha}$ and $f \in L^{p}\left(\omega d \mu_{\alpha}\right)$. If $\left\langle\ell_{k}^{\alpha}, f\right\rangle=0$ for all $k \in \mathbb{N}^{d}$ then $f=0$.

Proof. Apply the arguments from the proof of Corollary 3.8.

Let $1 \leq p<\infty$ and $\omega \in A_{p}^{\alpha}$. Given $f \in L^{p}\left(\omega d \mu_{\alpha}\right)$ we define its heatdiffusion integral by

$$
g^{\alpha}(t, x)=\sum_{n=0}^{\infty} e^{-t(n+(|\alpha|+d) / 2)} \sum_{|k|=n}\left\langle\ell_{k}^{\alpha}, f\right\rangle \ell_{k}^{\alpha}(x), \quad t>0 .
$$

The above series converges, since by (13) and Lemma 4.2,

$$
\begin{aligned}
& \sum_{n=0}^{\infty} e^{-t(n+(|\alpha|+d) / 2)} \sum_{|k|=n}\left|\left\langle\ell_{k}^{\alpha}, f\right\rangle\right|\left|\ell_{k}^{\alpha}(x)\right| \\
& \leq c \sum_{n=0}^{\infty} e^{-t(n+(|\alpha|+d) / 2)}(2 n+\|\alpha\|+1)^{\delta d+\|\alpha\| d / 2} n^{d}<\infty .
\end{aligned}
$$

To obtain an integral form of $g^{\alpha}(t, x)$ we write

$$
\begin{aligned}
g^{\alpha}(t, x) & =\sum_{n=0}^{\infty} e^{-t(n+(|\alpha|+d) / 2)} \sum_{|k|=n} \ell_{k}^{\alpha}(x) \int_{\mathbb{R}_{+}^{d}} \ell_{k}^{\alpha}(y) f(y) \mu_{\alpha}(d y) \\
& =\int_{\mathbb{R}_{+}^{d}}\left(\sum_{n=0}^{\infty} e^{-t(n+(|\alpha|+d) / 2)} \sum_{|k|=n} \ell_{k}^{\alpha}(x) \ell_{k}^{\alpha}(y)\right) f(y) \mu_{\alpha}(d y) \\
& =\int_{\mathbb{R}_{+}^{d}} G_{t}^{\alpha}(x, y) f(y) \mu_{\alpha}(d y) .
\end{aligned}
$$

Interchanging the order of summation and integration is easily justified by (13) and Lemma 4.2 (see (21)).

The kernel $G_{t}^{\alpha}(x, y)$ may be computed explicitly since a proper generating formula is available [L, (4.17.6)]. The result is 


$$
\begin{aligned}
G_{t}^{\alpha}(x, y)= & \left(2 \sinh \frac{t}{2}\right)^{-d} \exp \left(-\frac{1}{2} \operatorname{coth} \frac{t}{2} \sum_{i=1}^{d}\left(x_{i}+y_{i}\right)\right) \\
& \cdot \prod_{i=1}^{d}\left(\sqrt{x_{i} y_{i}}\right)^{-\alpha_{i}} I_{\alpha_{i}}\left(\frac{\sqrt{x_{i} y_{i}}}{\sinh (t / 2)}\right),
\end{aligned}
$$

where $x, y \in \mathbb{R}_{+}^{d}$ and $I_{a}(s)=i^{-a} J_{a}(i s)$ is the Bessel function of an imaginary argument (cf. [L]). In particular, it follows that $G_{t}^{\alpha}(x, y)$ is positive on $\mathbb{R}_{+}^{d} \times \mathbb{R}_{+}^{d}$.

Proposition 4.5. Let $1 \leq p<\infty, \omega \in A_{p}^{\alpha}$ and $f \in L^{p}\left(\omega d \mu_{\alpha}\right)$. The heat-diffusion integral $g^{\alpha}(t, x)$ of $f$ is a $C^{\infty}$ function on $\mathbb{R}_{+} \times \mathbb{R}_{+}^{d}$. Moreover, it satisfies

$$
\left(L_{x}-\partial / \partial t\right) g^{\alpha}(t, x)=0 .
$$

Proof. Since $\sum_{|k|=n}\left|\left\langle\ell_{k}^{\alpha}, f\right\rangle \ell_{k}^{\alpha}(x)\right|$ grows polynomially in $n$, uniformly with respect to $x$ (see $(21)$ ), we may differentiate in $t$ term by term the series defining $g^{\alpha}(t, x)$. The result is

$$
\begin{aligned}
\frac{\partial^{m}}{\partial t^{m}} g^{\alpha}(t, x)= & \sum_{n=0}^{\infty}(-1)^{m}\left(n+\frac{|\alpha|+d}{2}\right)^{m} \\
& \cdot e^{-t(n+(|\alpha|+d) / 2)} \sum_{|k|=n}\left\langle\ell_{k}^{\alpha}, f\right\rangle \ell_{k}^{\alpha}(x),
\end{aligned}
$$

RHS being continuous since the series converges almost uniformly in $(t, x)$.

Using the formula (cf. [L, (4.18.6)])

$$
\frac{\partial}{\partial x_{j}} \ell_{k}^{\alpha}(x)=-\sqrt{k_{j}} \ell_{k-e_{j}}^{\alpha+e_{j}}(x)-\frac{1}{2} \ell_{k}^{\alpha}(x), \quad k_{j}>0,
$$

together with (13) we obtain

$$
\left|\frac{\partial}{\partial x_{j}} \ell_{k}^{\alpha}(x)\right| \leq c(2|k|+\|\alpha\|+1)^{\|\alpha\| / 2+1} .
$$

Thus $\sum_{|k|=n}\left|\left\langle\ell_{k}^{\alpha}, f\right\rangle \partial_{x_{j}} \ell_{k}^{\alpha}(x)\right|$ grows polynomially in $n$, uniformly with respect to $x$. Therefore we may differentiate in $x_{j}$ term by term the series in (23), the result being a continuous function since the convergence is almost uniform in $(t, x)$. The same arguments apply to higher derivatives, so $g^{\alpha}(t, x)$ is smooth on $\mathbb{R}_{+} \times \mathbb{R}_{+}^{d}$.

The heat equation (22) is easily verified by differentiating term by term the series of $g^{\alpha}(t, x)$.

Denote by $M_{\mathrm{s}}^{\alpha}$ the strong maximal function in $\mathbb{R}_{+}^{d}$ with respect to the measure $\mu_{\alpha}$, i.e. given $f \in L_{\text {loc }}^{1}\left(\mathbb{R}_{+}^{d}, d \mu_{\alpha}\right)$ we have 


$$
M_{\mathrm{s}}^{\alpha} f(x)=\sup _{x \in H \in \mathcal{H}} \frac{1}{\mu_{\alpha}(H)} \int_{H}|f(y)| \mu_{\alpha}(d y),
$$

where $\mathcal{H}$ is the family of all "rectangles" in $\mathbb{R}_{+}^{d}$ with sides parallel to the coordinate axes.

For $1 \leq p<\infty$ we denote by $\left(A_{p}^{\alpha}\right)^{*}=A_{p}^{*}\left(\mathbb{R}_{+}^{d}, d \mu_{\alpha}\right)$ the strong $A_{p}$ class of weights in $\mathbb{R}_{+}^{d}$ with respect to the measure $\mu_{\alpha}$. More precisely, $\left(A_{p}^{\alpha}\right)^{*}$ consists of those functions from $A_{p}^{\alpha}$ which satisfy the condition (14) if $p>1$, or (15) if $p=1$, with the supremum taken over $\mathcal{H}$.

We note that if $p>1$ then $\omega \in\left(A_{p}^{\alpha}\right)^{*}$ if and only if $M_{\mathrm{S}}^{\alpha}$ is bounded on $L^{p}\left(\mathbb{R}_{+}^{d}, \omega d \mu_{\alpha}\right)$. This seems to be well known and follows by an adaptation of the proof for the Lebesgue measure case, which may be found for instance in $[\mathrm{GR}]$.

Theorem 4.6. Let $1<p<\infty, \omega \in\left(A_{p}^{\alpha}\right)^{*}$ and $f \in L^{p}\left(\omega d \mu_{\alpha}\right)$. Let $g^{\alpha}(t, x)$ be the heat-diffusion integral of $f$. Then

(a) $\sup _{t>0}\left|g^{\alpha}(t, x)\right| \leq C M_{\mathrm{s}}^{\alpha} f(x), x \in \mathbb{R}_{+}^{d}$;

(b) $\left\|g^{\alpha}(t, \cdot)\right\|_{L^{p}\left(\omega d \mu_{\alpha}\right)} \leq C \exp (-t(|\alpha|+d) / 2)\|f\|_{L^{p}\left(\omega d \mu_{\alpha}\right)}$;

(c) $\left\|g^{\alpha}(t, \cdot)-f\right\|_{L^{p}\left(\omega d \mu_{\alpha}\right)} \rightarrow 0, t \rightarrow 0^{+}$;

(d) $g^{\alpha}(t, x) \rightarrow f(x)$ a.e., $t \rightarrow 0^{+}$.

Moreover, the family $\left\{T_{t}^{\alpha}\right\}_{t>0}, T_{t}^{\alpha} f(x)=g^{\alpha}(t, x)$, is a strongly continuous and uniformly bounded semigroup of operators on $L^{p}\left(\omega d \mu_{\alpha}\right)$.

To prove the theorem we will need a multi-dimensional analogue of a result used by Muckenhoupt. The proof is a straightforward modification of that in [Mu1] and therefore is omitted.

LEMma 4.7. Let $\mu$ be a positive, absolutely continuous measure on $\mathbb{R}_{+}^{d}$. Assume that $f$ is a measurable function on $\mathbb{R}_{+}^{d}, g \in L^{1}\left(\mathbb{R}_{+}^{d}, d \mu\right), g \geq 0$ and $g(y)=g_{1}\left(y_{1}\right) \ldots g_{d}\left(y_{d}\right), y \in \mathbb{R}_{+}^{d}$. Suppose also that for some $x \in \mathbb{R}_{+}^{d}$ each $g_{i}(\cdot)$ is increasing for $y_{i}<x_{i}$ and decreasing for $y_{i}>x_{i}$. Then

$$
\int_{\mathbb{R}_{+}^{d}}|f(y)| g(y) \mu(d y) \leq\|g\|_{L^{1}(d \mu)} M_{\mathrm{S}}^{\mu} f(x),
$$

where $M_{\mathrm{s}}^{\mu}$ denotes the strong maximal function associated with the measure $\mu$.

Proof of Theorem 4.6. We have $G_{t}^{\alpha}(x, y)=\prod_{i=1}^{d} G_{t}^{\alpha_{i}}\left(x_{i}, y_{i}\right)$, where $G_{t}^{\alpha_{i}}\left(x_{i}, y_{i}\right) \geq 0$. By [St, Lemma 2.2] there exists a function $K(t, x, y)=$ $\prod_{i=1}^{d} K_{i}\left(t, x_{i}, y_{i}\right)$ with the following properties:

(i) $G_{t}^{\alpha_{i}}\left(x_{i}, y_{i}\right) \leq \exp \left(-t\left(\alpha_{i}+1\right) / 2\right) K_{i}\left(t, x_{i}, y_{i}\right), i=1, \ldots, d$;

(ii) for each $t>0$ and $x_{i}>0, K_{i}\left(t, x_{i}, y_{i}\right)$ as a function of $y_{i}$ is increasing on $\left[0, x_{i}\right]$ and decreasing on $\left[x_{i}, \infty\right)$;

(iii) $\int_{0}^{\infty} K_{i}\left(t, x_{i}, y_{i}\right) y_{i}{ }^{\alpha_{i}} d y_{i} \leq C$ independently of $x_{i}$ and $t>0$. 
Thus by Lemma 4.7,

$$
\left|g^{\alpha}(t, x)\right| \leq C e^{-t(|\alpha|+d) / 2} M_{\mathrm{s}}^{\alpha} f(x), \quad x \in \mathbb{R}_{+}^{d},
$$

and hence (a) and (b) follow. (c) and (d) are justified by standard arguments with the aid of (a), (b) and Lemma 4.3.

The semigroup property is easily verified to hold for any $\ell_{k}^{\alpha}$; hence by (b) and Lemma 4.3 it holds for all $f \in L^{p}\left(\omega d \mu_{\alpha}\right)$. Strong continuity follows by standard reasoning, similarly to (c).

Proposition 4.8. Let $1 \leq p, q<\infty, \omega \in A_{p}^{\alpha}, \nu \in A_{q}^{\alpha}$ and $f \in$ $L^{p}\left(\omega d \mu_{\alpha}\right)$. Then

$$
\left\|T_{t}^{\alpha} f\right\|_{L^{q}\left(\nu d \mu_{\alpha}\right)} \leq C(t)\|f\|_{L^{p}\left(\omega d \mu_{\alpha}\right)},
$$

where $C(t), t>0$, is a continuous and decreasing function of $t$ that vanishes at infinity.

Proof. Using Lemmas 4.1 and 4.2 we write

$$
\begin{aligned}
\left\|T_{t}^{\alpha} f\right\|_{L^{q}\left(\nu d \mu_{\alpha}\right)} & \leq \sum_{n=0}^{\infty} e^{-t(n+(d+|\alpha|) / 2)} \sum_{|k|=n}\left|\left\langle\ell_{k}^{\alpha}, f\right\rangle\right|\left\|\ell_{k}^{\alpha}\right\|_{L^{q}\left(\nu d \mu_{\alpha}\right)} \\
& \leq c\left(\sum_{n=0}^{\infty} e^{-t(n+(d+|\alpha|) / 2)}(2 n+\|\alpha\|+1)^{2 \delta d} n^{d}\right)\|f\|_{L^{p}\left(\omega d \mu_{\alpha}\right)} .
\end{aligned}
$$

Corollary 4.9. Let $1 \leq p<\infty$ and $\omega \in A_{p}^{\alpha}$. The family $\left\{T_{t}^{\alpha}\right\}_{t>0}$ is a strongly continuous semigroup of operators on $L^{p}\left(\omega d \mu_{\alpha}\right)$ (note that the continuity at $0^{+}$is not postulated here).

We now pass to Poisson integrals. Let $1 \leq p<\infty$ and $\omega \in A_{p}^{\alpha}$. Given $f \in L^{p}\left(\omega d \mu_{\alpha}\right)$ we define its Poisson integral by

$$
f^{\alpha}(t, x)=\sum_{n=0}^{\infty} e^{-t \sqrt{n+(|\alpha|+d) / 2}} \sum_{|k|=n}\left\langle\ell_{k}^{\alpha}, f\right\rangle \ell_{k}^{\alpha}(x), \quad t>0 .
$$

The above series converges (see (21)). Using (10) we obtain an integral form of $f^{\alpha}(t, x)$ :

$$
\begin{aligned}
f^{\alpha}(t, x)= & \sum_{n=0}^{\infty} \frac{t}{\sqrt{4 \pi}} \int_{0}^{\infty} e^{-s(n+(|\alpha|+d) / 2)} s^{-3 / 2} e^{-t^{2} /(4 s)} d s \\
& \cdot \sum_{|k|=n} \ell_{k}^{\alpha}(x) \int_{\mathbb{R}_{+}^{d}} \ell_{k}^{\alpha}(y) f(y) \mu_{\alpha}(d y) \\
= & \int_{\mathbb{R}_{+}^{d}} P_{t}^{\alpha}(x, y) f(y) \mu_{\alpha}(d y),
\end{aligned}
$$


where

$$
P_{t}^{\alpha}(x, y)=\frac{t}{\sqrt{4 \pi}} \int_{0}^{\infty} G_{s}^{\alpha}(x, y) s^{-3 / 2} e^{-t^{2} /(4 s)} d s .
$$

We also have the subordination formula

$$
f^{\alpha}(t, x)=\frac{t}{\sqrt{4 \pi}} \int_{0}^{\infty} g^{\alpha}(s, x) s^{-3 / 2} e^{-t^{2} /(4 s)} d s, \quad t>0 .
$$

Interchanging the order of integration and summation above is justified by (13) and Lemma 4.2.

Proposition 4.10. Let $1 \leq p<\infty, \omega \in A_{p}^{\alpha}$ and $f \in L^{p}\left(\omega d \mu_{\alpha}\right)$. The Poisson integral $f^{\alpha}(t, x)$ of $f$ is a $C^{\infty}$ function on $\mathbb{R}_{+} \times \mathbb{R}_{+}^{d}$. Moreover, it satisfies

$$
\left(L_{x}+\partial^{2} / \partial t^{2}\right) f^{\alpha}(t, x)=0 .
$$

Proof. Apply the arguments from the proof of Proposition 4.5.

Theorem 4.11. Assume that $1<p<\infty, \omega \in\left(A_{p}^{\alpha}\right)^{*}$ and $f \in L^{p}\left(\omega d \mu_{\alpha}\right)$. Let $f^{\alpha}(t, x)$ be the Poisson integral of $f$. Then

(a) $\sup _{t>0}\left|f^{\alpha}(t, x)\right| \leq C M_{\mathrm{s}}^{\alpha} f(x), \quad x \in \mathbb{R}_{+}^{d}$;

(b) $\left\|f^{\alpha}(t, \cdot)\right\|_{L^{p}\left(\omega d \mu_{\alpha}\right)} \leq C \exp (-t \sqrt{(|\alpha|+d) / 2})\|f\|_{L^{p}\left(\omega d \mu_{\alpha}\right)}$;

(c) $\left\|f^{\alpha}(t, \cdot)-f\right\|_{L^{p}\left(\omega d \mu_{\alpha}\right)} \rightarrow 0, t \rightarrow 0^{+}$;

(d) $f^{\alpha}(t, x) \rightarrow f(x)$ a.e., $t \rightarrow 0^{+}$.

Moreover, the family $\left\{P_{t}^{\alpha}\right\}_{t>0}, P_{t}^{\alpha} f(x)=f^{\alpha}(t, x)$, is a strongly continuous and uniformly bounded semigroup of operators on $L^{p}\left(\mathbb{R}_{+}^{d}, \omega d \mu_{\alpha}\right)$.

Proof. Using the subordination formula (25) and (24) we get

$$
\begin{aligned}
\left|f^{\alpha}(t, x)\right| & \leq c M_{\mathrm{s}}^{\alpha} f(x) \int_{0}^{\infty} e^{-s(|\alpha|+d) / 2} \frac{t}{\sqrt{4 \pi}} s^{-3 / 2} e^{-t^{2} /(4 s)} d s \\
& =c e^{-t \sqrt{(|\alpha|+d) / 2}} M_{\mathrm{s}}^{\alpha} f(x) .
\end{aligned}
$$

This shows (a) and (b). The rest of the proof is similar to the proof of Theorem 4.6. Then

Proposition 4.12. Let $1 \leq p, q<\infty, \omega \in A_{p}^{\alpha}, \nu \in A_{q}^{\alpha}$ and $f \in L^{p}\left(\omega d \mu_{\alpha}\right)$.

$$
\left\|P_{t}^{\alpha} f\right\|_{L^{q}\left(\nu d \mu_{\alpha}\right)} \leq C(t)\|f\|_{L^{p}\left(\omega d \mu_{\alpha}\right)}
$$

$C(t), t>0$, being a continuous and decreasing function of that vanishes at infinity.

Proof. Argue as in the proof of Proposition 4.8.

Corollary 4.13. Let $1 \leq p<\infty$ and $\omega \in A_{p}^{\alpha}$. The family $\left\{P_{t}^{\alpha}\right\}_{t>0}$ is a strongly continuous semigroup of operators on $L^{p}\left(\mathbb{R}_{+}^{d}, \omega d \mu_{\alpha}\right)$. 
REMARK 4.14. A large part of the results of this section are valid for the space $L^{\infty}\left(\mathbb{R}_{+}^{d}\right)$. More precisely, Lemmas 4.1 and 4.2 and Propositions 4.5 and 4.10 remain valid if we replace $L^{p}\left(\omega d \mu_{\alpha}\right)$ by $L^{\infty}$. Moreover, Theorems 4.6 and 4.11, except (c) and (d), also remain valid with $L^{\infty}$ replacing $L^{p}\left(\omega d \mu_{\alpha}\right)$. Concerning (c) and (d), we have $\left\|g^{\alpha}(t, \cdot)-f\right\|_{\infty} \rightarrow 0$ and $\left\|f^{\alpha}(t, \cdot)-f\right\|_{\infty} \rightarrow 0$ as $t \rightarrow 0^{+}$, but only for $f \in C_{0}\left(\mathbb{R}_{+}^{d}\right)$.

REMARK 4.15. If $1 \leq p \leq \infty$ and $f \in L^{p}\left(\mathbb{R}_{+}^{d}, d \mu_{\alpha}\right)$ (the case $\omega \equiv 1$ ) then Theorem 4.6(b) holds with the coefficient $C \exp (-t(|\alpha|+d) / 2)$ replaced by $(\cosh (t / 2))^{-(|\alpha|+d)}$ (cf. computations in [St]) and Theorem 4.11(b) holds with $C$ dropped. This means, in particular, that $\left\{T_{t}^{\alpha}\right\}_{t>0}$ and $\left\{P_{t}^{\alpha}\right\}_{t>0}$ are semigroups of contractions on $L^{p}\left(\mathbb{R}_{+}^{d}, d \mu_{\alpha}\right), 1 \leq p \leq \infty$. Note also that the above together with Lemma 4.3 implies $L^{p}$ convergence (part (c) in both theorems) for $f \in L^{p}\left(d \mu_{\alpha}\right), 1 \leq p<\infty$, which for $p=1$ could not be concluded earlier.

REMARK 4.16. In dimension one we have $M_{\mathrm{s}}^{\alpha}=M^{\alpha}$ (here $M^{\alpha}$ denotes the Hardy-Littlewood maximal function in $\mathbb{R}_{+}$with respect to the measure $\left.\mu_{\alpha}\right)$. Consequently, Theorems 4.6(d) and 4.11(d) hold with $p=1$ admitted.

5. Laguerre expansions; system $\left\{\varphi_{k}^{\alpha}\right\}$. Let $k=\left(k_{1}, \ldots, k_{d}\right) \in \mathbb{N}^{d}$ and $\alpha=\left(\alpha_{1}, \ldots, \alpha_{d}\right) \in(-1, \infty)^{d}$ be multi-indices. The Laguerre function $\varphi_{k}^{\alpha}$ on $\mathbb{R}_{+}^{d}$ is defined as

$$
\varphi_{k}^{\alpha}(x)=\varphi_{k_{1}}^{\alpha_{1}}\left(x_{1}\right) \cdot \ldots \cdot \varphi_{k_{d}}^{\alpha_{d}}\left(x_{d}\right), \quad x=\left(x_{1}, \ldots, x_{d}\right) \in \mathbb{R}_{+}^{d},
$$

where $\varphi_{k_{i}}^{\alpha_{i}}$ are the one-dimensional Laguerre functions given by

$\varphi_{k_{i}}^{\alpha_{i}}\left(x_{i}\right)=\left(\frac{2 \Gamma\left(k_{i}+1\right)}{\Gamma\left(k_{i}+\alpha_{i}+1\right)}\right)^{1 / 2} L_{k_{i}}^{\alpha_{i}}\left(x_{i}^{2}\right) x_{i}^{\alpha_{i}+1 / 2} e^{-x_{i}^{2} / 2}, \quad x_{i}>0, i=1, \ldots, d$.

Note that only for $\alpha_{i} \geq-1 / 2, i=1, \ldots, d$, do the functions $\varphi_{k}^{\alpha}$ belong to all $L^{p}$ spaces on $\mathbb{R}_{+}^{d}, 1 \leq p<\infty$. Therefore we assume throughout this section that $\alpha \in[-1 / 2, \infty)^{d}$.

Each $\varphi_{k}^{\alpha}$ is an eigenfunction of the differential operator

$$
L=\Delta-|x|^{2}-\sum_{i=1}^{d} \frac{1}{x_{i}^{2}}\left(\alpha_{i}^{2}-\frac{1}{4}\right),
$$

the corresponding eigenvalue being $-(4|k|+2|\alpha|+2 d)$. The operator $-L$ is positive and symmetric in $L^{2}\left(\mathbb{R}_{+}^{d}, d x\right)$. Furthermore, the system $\left\{\varphi_{k}^{\alpha}\right.$ : $\left.k \in \mathbb{N}^{d}\right\}$ is an orthonormal basis in $L^{2}\left(\mathbb{R}_{+}^{d}, d x\right)$.

The following estimate of $\varphi_{k}^{\alpha}$ is essential for our considerations:

$$
\left|\varphi_{k}^{\alpha}(x)\right| \leq c \prod_{i=1}^{d} \Psi_{k_{i}}^{\alpha_{i}}\left(x_{i}\right), \quad x \in \mathbb{R}_{+}^{d},
$$


where

$$
\Psi_{k_{i}}^{\alpha_{i}}\left(x_{i}\right)= \begin{cases}1, & 0<x_{i} \leq 4\left(2 k_{i}+\alpha_{i}+1\right), \\ \exp \left(-\gamma x_{i}\right), & x_{i}>4\left(2 k_{i}+\alpha_{i}+1\right) .\end{cases}
$$

Here $c$ and $\gamma$ are independent of $k$ and $x$. Similarly to (13), the above estimate follows by Muckenhoupt's generalization of the estimates proved by Askey and Wainger.

In this section we denote by $A_{p}=A_{p}\left(\mathbb{R}_{+}^{d}, d x\right), 1 \leq p<\infty$, the class of $A_{p}$ weights on $\mathbb{R}_{+}^{d}$ with respect to the Lebesgue measure $d x$.

Let $1 \leq p<\infty$ and $\omega \in A_{p}$. The lemmas below are analogues of Lemmas 4.1-4.3. Their proofs are almost the same as for the system $\left\{\ell_{k}^{\alpha}\right\}$, the only essential difference being the estimate for Laguerre functions (27).

LEMmA 5.1. There exists a constant $c$ independent of $k \in \mathbb{N}^{d}$ such that

$$
\left\|\varphi_{k}^{\alpha}\right\|_{L^{p}(\omega)} \leq c(2|k|+|\alpha|+d)^{d}
$$

Moreover, the Fourier-Laguerre coefficients $\left\langle\varphi_{k}^{\alpha}, f\right\rangle=\int_{\mathbb{R}_{+}^{d}} \varphi_{k}^{\alpha}(x) f(x) d x$ exist for $f \in L^{p}(\omega)$ and they satisfy

$$
\left|\left\langle\varphi_{k}^{\alpha}, f\right\rangle\right| \leq C(2|k|+|\alpha|+d)^{d}\|f\|_{L^{p}(\omega)},
$$

with a constant $C$ independent of $k \in \mathbb{N}^{d}$.

LEMMA 5.2. The subspace spanned by $\left\{\varphi_{k}^{\alpha}: k \in \mathbb{N}^{d}\right\}$ is dense in $L^{p}(\omega)$ and in $C_{0}\left(\mathbb{R}_{+}^{d}\right)$ with $\|\cdot\|_{\infty}$ norm.

Corollary 5.3. Let $f \in L^{p}(\omega)$. If $\left\langle\varphi_{k}^{\alpha}, f\right\rangle=0$ for all $k \in \mathbb{N}^{d}$ then $f=0$.

Assume that $1 \leq p<\infty$ and $\omega \in A_{p}$. Given $f \in L^{p}(\omega)$ we define its heat-diffusion integral by

$$
g^{\alpha}(t, x)=\sum_{n=0}^{\infty} e^{-t(4 n+2|\alpha|+2 d)} \sum_{|k|=n}\left\langle\varphi_{k}^{\alpha}, f\right\rangle \varphi_{k}^{\alpha}(x), \quad t>0 .
$$

The above series converges by (27) and Lemma 5.1. Similarly to the case of the system $\left\{\ell_{k}^{\alpha}\right\}$ we obtain an integral form of $g^{\alpha}(t, x)$ :

$$
g^{\alpha}(t, x)=\int_{\mathbb{R}_{+}^{d}} G_{t}^{\alpha}(x, y) f(y) d y
$$

The kernel $G_{t}^{\alpha}(x, y)$ may be computed by using the formula [L, (4.17.6)] to be

$$
\begin{aligned}
G_{t}^{\alpha}(x, y)= & (\sinh 2 t)^{-d} \exp \left(-\frac{1}{2} \operatorname{coth}(2 t)\left(|x|^{2}+|y|^{2}\right)\right) \\
& \cdot \prod_{i=1}^{d} \sqrt{x_{i} y_{i}} I_{\alpha_{i}}\left(\frac{x_{i} y_{i}}{\sinh (2 t)}\right) .
\end{aligned}
$$

Note that $G_{t}^{\alpha}(x, y)$ is positive on $\mathbb{R}_{+}^{d} \times \mathbb{R}_{+}^{d}$. 
Proposition 5.4. Let $1 \leq p<\infty, \omega \in A_{p}$ and $f \in L^{p}(\omega)$. The heatdiffusion integral $g^{\alpha}(t, x)$ of $f$ is a $C^{\infty}$ function on $\mathbb{R}_{+} \times \mathbb{R}_{+}^{d}$. Moreover, it satisfies

$$
\left(L_{x}-\partial / \partial t\right) g^{\alpha}(t, x)=0 .
$$

Proof. The conclusion follows by the reasoning from the proof of Proposition 4.5 provided we have a proper estimate of $\partial^{\beta} \varphi_{k}^{\alpha}$ at our disposal. Since (cf. [L, (4.18.6)])

$$
\frac{\partial}{\partial x_{j}} \varphi_{k}^{\alpha}(x)=-2 \sqrt{k_{j}} \varphi_{k-e_{j}}^{\alpha+e_{j}}(x)+\left(\frac{2 \alpha_{j}+1}{2 x_{j}}-x_{j}\right) \varphi_{k}^{\alpha}(x), \quad k_{j}>0,
$$

the estimate (27) gives what is needed:

$$
\left|\frac{\partial}{\partial x_{j}} \varphi_{k}^{\alpha}(x)\right| \leq c\left(\varepsilon+\varepsilon^{-1}\right) \sqrt{|k|}, \quad x \in\left[\varepsilon, \varepsilon^{-1}\right]^{d},
$$

for any $\varepsilon \in(0,1)$; similarly for higher derivatives.

Denote by $M_{+}$the (centered) Hardy-Littlewood maximal function in $\mathbb{R}_{+}^{d}$, i.e.

$$
M_{+} f(x)=\sup \frac{1}{|Q|} \int_{Q}|f(y)| d y, \quad x \in \mathbb{R}_{+}^{d},
$$

where the supremum is taken over all sets of the form $Q=\widetilde{Q} \cap \mathbb{R}_{+}^{d}$, and $\widetilde{Q}$ are cubes (with sides parallel to the coordinate axes) in $\mathbb{R}^{d}$ centered at $x$.

Theorem 5.5. Assume that $1 \leq p<\infty, \omega \in A_{p}$ and $f \in L^{p}(\omega)$. Let $g^{\alpha}(t, x)$ be the heat-diffusion integral of $f$. Then

(a) $\left|g^{\alpha}(t, x)\right| \leq C M_{+} f(x), x \in \mathbb{R}_{+}^{d}$;

(b) $\left\|g^{\alpha}(t, \cdot)\right\|_{L^{p}(\omega)} \leq C\|f\|_{L^{p}(\omega)}$;

(c) $\left\|g^{\alpha}(t, \cdot)-f\right\|_{L^{p}(\omega)} \rightarrow 0, t \rightarrow 0^{+}$;

(d) $g^{\alpha}(t, x) \rightarrow f(x)$ a.e., $t \rightarrow 0^{+}$.

Moreover, the family $\left\{T_{t}^{\alpha}\right\}_{t>0}, T_{t}^{\alpha} f(x)=g^{\alpha}(t, x)$, is a strongly continuous and uniformly bounded semigroup of operators on $L^{p}(\omega)$.

Proof. Let $W(x)=(4 \pi)^{-d / 2} \exp \left(-|x|^{2} / 4\right)$ be the Gauss-Weierstrass kernel in $\mathbb{R}^{d}$ and let $W_{\varepsilon}(\cdot)=\varepsilon^{-d} W(\cdot / \varepsilon)$ be its $\varepsilon$-dilation. We claim that there exists a constant $C$ depending only on $\alpha$ such that

$$
G_{t}^{\alpha}(x, y) \leq C(2 \pi)^{d / 2} W_{\sinh (2 t) / 2}(y-x) .
$$

This is proved by using the explicit formula (28) for $G_{t}^{\alpha}(x, y)$ and the following estimate for $I_{\beta}, \beta \geq-1 / 2$ :

$$
c^{-1} \Lambda(s) \leq I_{\beta}(s) \leq c \Lambda(s), \quad s>0,
$$


with $c$ depending only on $\beta$ and the function $\Lambda$ defined by

$$
\Lambda(s)= \begin{cases}s^{\beta}, & 0<s \leq 1 \\ s^{-1 / 2} e^{s}, & 1<s<\infty\end{cases}
$$

Let $\tilde{f}$ be an extension of $f$ to $\mathbb{R}^{d}$ such that $\tilde{f}(x)=0$ for $x \notin \mathbb{R}_{+}^{d}$. By (29) we have $\left|g^{\alpha}(t, x)\right| \leq c|\widetilde{f}| * W_{\sinh (2 t) / 2}(x)$ and hence (a) and (b) follow by the $\mathbb{R}^{d}$ versions of Lemma 3.2 and Remark 3.3. This together with Lemma 5.2 justifies (c) and (d) in a standard manner.

The semigroup property is immediately verified for any $\varphi_{k}^{\alpha}$, hence by (b) and Lemma 5.2 it holds for all $f \in L^{p}(\omega)$. Strong continuity follows by standard arguments, with the aid of (b) and Lemma 5.2.

Let us pass to Poisson integrals. Assume that $1 \leq p<\infty$ and $\omega \in A_{p}$. Given $f \in L^{p}(\omega)$ we define its Poisson integral by

$$
f^{\alpha}(t, x)=\sum_{n=0}^{\infty} e^{-t \sqrt{4 n+2|\alpha|+2 d}} \sum_{|k|=n}\left\langle\varphi_{k}^{\alpha}, f\right\rangle \varphi_{k}^{\alpha}(x), \quad t>0 .
$$

The above series converges by (27) and Lemma 5.1. An integral form of $f^{\alpha}(t, x)$ as well as the corresponding subordination formula are obtained by (10). Applying arguments from the proof of Proposition 5.4 we get

Proposition 5.6. Let $1 \leq p<\infty, \omega \in A_{p}$ and $f \in L^{p}(\omega)$. The Poisson integral $f^{\alpha}(t, x)$ of $f$ is a $C^{\infty}$ function on $\mathbb{R}_{+} \times \mathbb{R}_{+}^{d}$. Moreover, it satisfies

$$
\left(L_{x}+\partial^{2} / \partial t^{2}\right) f^{\alpha}(t, x)=0 .
$$

The main result on Poisson integrals reads as follows.

Theorem 5.7. Assume that $1 \leq p<\infty, \omega \in A_{p}$ and $f \in L^{p}(\omega)$. Let $f^{\alpha}(t, x)$ be the Poisson integral of $f$. Then

(a) $\left|f^{\alpha}(t, x)\right| \leq C M_{+} f(x), x \in \mathbb{R}_{+}^{d}$;

(b) $\left\|f^{\alpha}(t, \cdot)\right\|_{L^{p}(\omega)} \leq C\|f\|_{L^{p}(\omega)}$;

(c) $\left\|f^{\alpha}(t, \cdot)-f\right\|_{L^{p}(\omega)} \rightarrow 0, t \rightarrow 0^{+}$;

(d) $f^{\alpha}(t, x) \rightarrow f(x)$ a.e., $t \rightarrow 0^{+}$.

Moreover, the family $\left\{P_{t}^{\alpha}\right\}_{t>0}, P_{t}^{\alpha} f(x)=f^{\alpha}(t, x)$, is a strongly continuous and uniformly bounded semigroup of operators on $L^{p}(\omega)$.

Proof. Items (a) and (b) follow by Theorem 5.5 and the subordination formula (see the proof of Theorem 3.12). The rest is justified as in the case of the heat-diffusion integrals.

REMARK 5.8. A large part of the results of this section are valid for the space $L^{\infty}\left(\mathbb{R}_{+}^{d}\right)$. More precisely, Lemma 5.1, Proposition 5.4 and Proposition 5.6 remain valid if we replace $L^{p}(\omega)$ by $L^{\infty}$. Further, Theorem 5.5 and Theorem 5.7, except (c) and (d), also remain valid with $L^{\infty}$ replacing $L^{p}(\omega)$. 
Concerning (c) and (d), we have $\left\|g^{\alpha}(t, \cdot)-f\right\|_{\infty} \rightarrow 0$ and $\left\|f^{\alpha}(t, \cdot)-f\right\|_{\infty} \rightarrow 0$ as $t \rightarrow 0^{+}$, but only for $f \in C_{0}\left(\mathbb{R}_{+}^{d}\right)$.

REMARK 5.9. Let $M_{\mathrm{S}}$ be the strong maximal function in $\mathbb{R}_{+}^{d}$ and denote by $A_{p}^{*}$ the strong $A_{p}$ class of weights in $\mathbb{R}_{+}^{d}$. Using [St, Lemma 4.2] and Lemma 4.7 one may obtain

$$
\left|g^{\alpha}(t, x)\right| \leq C e^{-2 t|\alpha \wedge 1 / 2|} M_{\mathrm{s}} f(x), \quad x \in \mathbb{R}_{+}^{d},
$$

with the notation $|\alpha \wedge 1 / 2|=\sum_{i=1}^{d} \min \left(\alpha_{i}, 1 / 2\right)$. Thus, when $1<p<\infty$ and $\omega \in A_{p}^{*}$, the constants $C$ in Theorems 5.5(b) and 5.7(b) may be replaced by $C e^{-2 t|\alpha \wedge 1 / 2|}$ and $C e^{-t \sqrt{2 \max \{|\alpha \wedge 1 / 2|, 0\}}}$, respectively. If $|\alpha \wedge 1 / 2|>0$ this gives the exponential decrease in $t$ at infinity.

REMARK 5.10. If $1 \leq p \leq \infty$ and $f \in L^{p}\left(\mathbb{R}_{+}^{d}\right)$ (the case $\omega \equiv 1$ ) then Theorem 5.5(b) holds with $C$ replaced by $C \exp (-2 t(|\alpha|+d))$ (cf. estimates in [St]) and Theorem 5.7(b) holds with $C \exp (-t \sqrt{2(|\alpha|+d)})$ instead of $C$.

6. Connection between Hermite and Laguerre expansions. In this section we briefly show how some results for Hermite semigroups may be transferred to the Laguerre setting. We will exploit the ideas of Dinger [Di], developed later in [GIT], for Hermite and Laguerre polynomial systems. The lemmas we shall use are straightforward modifications of those in [GIT] and therefore we provide no proofs here.

The key fact underlying the idea of transference is that if $\alpha$ has a special form, then the one-dimensional Laguerre functions $\ell_{k}^{\alpha}$ can be expressed by means of multi-dimensional Hermite functions. The following lemma makes this precise (cf. [Di]).

Lemma 6.1. Let $\ell_{k}^{\alpha}$ be the one-dimensional Laguerre function of type $\alpha$ with $\alpha=n / 2-1, n \in \mathbb{N} \backslash\{0\}$, and let $x \in \mathbb{R}^{n}$. Then we have the expansion

$$
\ell_{k}^{\alpha}\left(|x|^{2}\right)=\sum_{|r|=k} a_{r} h_{2 r}(x), \quad r=\left(r_{1}, \ldots, r_{n}\right) \in \mathbb{N}^{n} .
$$

Now, let $n=\left(n_{1}, \ldots, n_{d}\right) \in \mathbb{N}^{d}$ be a multi-index and define $x^{i}=$ $\left(x_{1}^{i}, \ldots, x_{n_{i}}^{i}\right) \in \mathbb{R}^{n_{i}}, i=1, \ldots, d$. We define the quadratic transformation $\phi: \mathbb{R}^{|n|} \rightarrow \mathbb{R}^{d}$ by

$$
\phi\left(x^{1}, \ldots, x^{d}\right)=\left(\left|x^{1}\right|^{2}, \ldots,\left|x^{d}\right|^{2}\right) .
$$

LEMMA 6.2. Let $\alpha=\left(\alpha_{1}, \ldots, \alpha_{d}\right)$ with $\alpha_{i}=n_{i} / 2-1$ and $n=\left(n_{1}, \ldots, n_{d}\right)$ $\in(\mathbb{N} \backslash\{0\})^{d}$. Given a weight $\omega$ in $\mathbb{R}_{+}^{d}$ and a measurable function $f$, the following holds:

$$
c(d, n) \int_{\mathbb{R}_{+}^{d}} f(y) \omega(y) y^{\alpha} d y=\int_{\mathbb{R}^{|n|}} f \circ \phi(x) \omega \circ \phi(x) d x,
$$

provided one of the integrals is absolutely convergent. 
Recall that $\mu_{\alpha}(d x)=x^{\alpha} d x$.

LEMMA 6.3. Let $\alpha, n$ and $\omega$ be as in Lemma 6.2, $p \in[1, \infty)$, and let $f$ be a fixed function in $L^{p}\left(\mathbb{R}_{+}^{d}, \omega d \mu_{\alpha}\right)$. Suppose that $T, \widetilde{T}$ are operators defined on $L^{p}\left(\mathbb{R}_{+}^{d}, \omega d \mu_{\alpha}\right)$ and $L^{p}\left(\mathbb{R}^{|n|}, \omega \circ \phi\right)$ respectively, satisfying $(T f)(\phi(x))=$ $\widetilde{T}(f \circ \phi)(x)$ for $x \in \mathbb{R}^{|n|}$. If

$$
\|\widetilde{T} f\|_{L^{p}\left(\mathbb{R}^{|n|}, \omega \circ \phi\right)} \leq C\|f\|_{L^{p}\left(\mathbb{R}^{|n|}, \omega \circ \phi\right)}
$$

then also

$$
\|T f\|_{L^{p}\left(\mathbb{R}_{+}^{d}, \omega d \mu_{\alpha}\right)} \leq C\|f\|_{L^{p}\left(\mathbb{R}_{+}^{d}, \omega d \mu_{\alpha}\right)}
$$

with the same constant $C$. Moreover, in the case $p=1$ an analogous statement is true for weighted weak type inequalities.

Let $\left\{T_{t}^{\alpha}\right\}$ and $\left\{P_{t}^{\alpha}\right\}$ be the heat-diffusion and Poisson semigroups associated with the Laguerre system $\left\{\ell_{k}^{\alpha}\right\}$. Denote by $\left\{T_{t}^{H}\right\}$ and $\left\{P_{t}^{H}\right\}$ the corresponding semigroups for the Hermite system $\left\{h_{k}\right\}$ (see Section 2 for the definition of $h_{k}$ and related facts).

LEMMA 6.4. Assume that $\alpha$ and $n$ are as in Lemma 6.2. Then for any $f \in \operatorname{lin}\left\{\ell_{k}^{\alpha}: k \in \mathbb{N}^{d}\right\}$ we have

$$
\left(T_{t}^{\alpha} f\right)(\phi(x))=T_{t / 2}^{H}(f \circ \phi)(x), \quad\left(P_{t}^{\alpha} f\right)(\phi(x))=P_{t / \sqrt{2}}^{H}(f \circ \phi)(x), \quad x \in \mathbb{R}^{|n|} .
$$

Let $A_{p}^{\alpha}=A_{p}\left(\mathbb{R}_{+}^{d}, d \mu_{\alpha}\right)$ be the class of weights from Section 4 . For $\alpha$ and $n$ as in Lemma 6.2 we define

$$
\widetilde{A}_{p}^{\alpha}=\left\{\omega \in A_{p}^{\alpha}: \omega \circ \phi \in A_{p}\left(\mathbb{R}^{|n|}\right)\right\} .
$$

The above class is considerably large. In fact, the following inclusion holds:

$$
\left(A_{p}^{\alpha}\right)^{*} \subset \widetilde{A}_{p}^{\alpha}, \quad 1 \leq p<\infty .
$$

We sketch a proof of this fact for $p>1$ (the same reasoning applies if $p=1$ ).

Let $Q \subset \mathbb{R}^{|n|}$ be a cube with sides parallel to the coordinate axes. We have $Q=Q_{1} \times \ldots \times Q_{d}$, where each $Q_{i}$ is a cube in $\mathbb{R}^{n_{i}}$. Denote by $S_{i}$ the smallest rectangle in polar coordinates in $\mathbb{R}^{n_{i}}$ that contains $Q_{i}$. Note that $\left|S_{i}\right|$ and $\left|Q_{i}\right|$ are comparable, with a constant independent of $Q_{i}$. Thus so are $|Q|$ and $|S|, S=S_{1} \times \ldots \times S_{d}$. Given a weight function $\omega$ in $\mathbb{R}^{d}$ we get

$$
\frac{1}{|Q|} \int_{Q} \omega \circ \phi(x) d x \leq c \frac{1}{|S|} \int_{S} \omega \circ \phi(x) d x=c \frac{1}{|\widetilde{S}|} \int_{\widetilde{S}} \omega \circ \phi(x) d x .
$$

Here $\widetilde{S}=\widetilde{S}_{1} \times \ldots \times \widetilde{S}_{d}$, with $\widetilde{S}_{i}$ being the radialization of $S_{i}$ in $\mathbb{R}^{n_{i}}$. The last equality in $(32)$ holds since $\omega \circ \phi$ is poly-radial on $\mathbb{R}^{n_{1}} \times \ldots \times \mathbb{R}^{n_{d}}$. Now, making a proper change of variables and integrating in polar coordinates on 
each $\mathbb{R}^{n_{i}}$ we obtain

$$
\frac{1}{|\widetilde{S}|} \int_{\widetilde{S}} \omega \circ \phi(x) d x=c \frac{1}{\mu_{\alpha}(\phi(\widetilde{S}))} \int_{\phi(\widetilde{S})} \omega(y) \mu_{\alpha}(d y) .
$$

Treating $(\omega \circ \phi)^{-p^{\prime} / p}$ similarly we conclude that

$$
\begin{aligned}
& {\left[\frac{1}{|Q|} \int_{Q} \omega \circ \phi(x) d x\right]\left[\frac{1}{|Q|} \int_{Q}(\omega \circ \phi(x))^{-p^{\prime} / p} d x\right]^{p / p^{\prime}}} \\
& \quad \leq c\left[\frac{1}{\mu_{\alpha}(\phi(\widetilde{S}))} \int_{\phi(\widetilde{S})} \omega(y) \mu_{\alpha}(d y)\right]\left[\frac{1}{\mu_{\alpha}(\phi(\widetilde{S}))} \int_{\phi(\widetilde{S})}(\omega(y))^{-p^{\prime} / p} \mu_{\alpha}(d y)\right]^{p / p^{\prime}},
\end{aligned}
$$

with $c$ independent of $Q$. Since $\phi(\widetilde{S})$ is a rectangle in $\mathbb{R}^{d}$, this clearly shows that $\omega \circ \phi \in A_{p}\left(\mathbb{R}^{|n|}\right)$ if only $\omega \in\left(A_{p}^{\alpha}\right)^{*}$. Hence (31) follows.

As a corollary of the above lemmas, Lemma 4.3 and the results for Hermite semigroups [StTo, Theorems 2.6 and 2.8], we obtain

TheOREM 6.5. Let $\alpha=\left(\alpha_{1}, \ldots, \alpha_{d}\right)$ with $\alpha_{i}=n_{i} / 2-1$ and $n_{i} \in \mathbb{N} \backslash\{0\}$. Assume that $\omega \in \widetilde{A}_{p}^{\alpha}$. Then the maximal operators

$$
T_{t}^{*} f=\sup _{t>0}\left|T_{t}^{\alpha} f\right| \quad \text { and } \quad P_{t}^{*} f=\sup _{t>0}\left|P_{t}^{\alpha} f\right|
$$

defined on $L^{p}\left(\omega d \mu_{\alpha}\right)$ are bounded if $1<p<\infty$, and weakly bounded if $p=1$. Moreover, the semigroups $\left\{T_{t}^{\alpha}\right\}$ and $\left\{P_{t}^{\alpha}\right\}$ are uniformly bounded on $L^{p}\left(\omega d \mu_{\alpha}\right), 1 \leq p<\infty$.

As a consequence of Theorem 6.5 and Lemma 4.3 we get

Corollary 6.6. Let $1 \leq p<\infty$ and $f \in L^{p}\left(\omega d \mu_{\alpha}\right)$. Under the assumptions of Theorem 6.5, we have

$$
T_{t}^{\alpha} f \rightarrow f, \quad P_{t}^{\alpha} f \rightarrow f, \quad t \rightarrow 0^{+},
$$

the convergence being both in $L^{p}\left(\omega d \mu_{\alpha}\right)$ and almost everywhere.

REMARK 6.7. When the multi-index $\alpha$ has a special form, Theorem 6.5 extends Theorems 4.6 and 4.11. This generalization is particularly significant in the case $p=1$, since weak boundedness of $T_{t}^{*}$ and $P_{t}^{*}$ seems to be a new result, even in the unweighted setting $(\omega \equiv 1)$ if $d>1$.

REMARK 6.8. Still another transference may be carried out from the special Hermite setting to the Laguerre setting based on the system $\left\{\psi_{k}^{\alpha}\right.$ : $k \in \mathbb{N}\}, \alpha>-1$. The functions $\psi_{k}^{\alpha}$ are defined by

$$
\begin{aligned}
\psi_{k}^{\alpha}(r) & =\left(\frac{\Gamma(k+1)}{2^{\alpha} \Gamma(k+\alpha+1)}\right)^{1 / 2} L_{k}^{\alpha}\left(\frac{r^{2}}{2}\right) \exp \left(-\frac{1}{4} r^{2}\right) \\
& =2^{-\alpha / 2} \ell_{k}^{\alpha}\left(\frac{r^{2}}{2}\right), \quad r>0,
\end{aligned}
$$


and they constitute an orthonormal basis in $L^{2}\left(\mathbb{R}_{+}, r^{2 \alpha+1} d r\right)$. If $f(z)=\widetilde{f}(r)$, $r=|z|$, is a radial function on $\mathbb{C}^{n}$, then its special Hermite expansion reduces to the Laguerre expansion of $\tilde{f}$ with respect to the system $\left\{\psi_{k}^{n-1}\right\}$ (see [Th2] for details). Using the results of Section 3 and the fact that if $\omega \in A_{p}\left(\mathbb{R}_{+}, r^{2 n-1} d r\right)$ then $\omega(|\cdot|) \in A_{p}\left(\mathbb{C}^{n}\right)$, which is justified similarly to (31), we obtain for $\left\{\psi_{k}^{n-1}\right\}$ conclusions analogous to those from Theorem 6.5.

REMark 6.9. To treat the system $\left\{\psi_{k}^{\alpha}\right\}$ also in higher dimensions and for all half-integer multi-indices $\alpha$ (i.e. such as in Theorem 6.5) one may use the transference from Hermite function expansions described in this section, but with the quadratic transformation (30) replaced by $\phi\left(x^{1}, \ldots, x^{d}\right)=$ $\left(\left|x^{1}\right|, \ldots,\left|x^{d}\right|\right)$. In particular an analogue of Theorem 6.5 follows.

Remark 6.10. Similar analysis to those from Sections 4 and 5 may be conducted for another Laguerre system $\left\{\mathscr{L}_{k}^{\alpha}: k \in \mathbb{N}^{d}\right\}$, defined by

$$
\mathscr{L}_{k}^{\alpha}(x)=\ell_{k}^{\alpha}(x) x^{\alpha / 2}, \quad x \in \mathbb{R}_{+}^{d} .
$$

The system $\left\{\mathscr{L}_{k}^{\alpha}\right\}$ is an orthonormal basis in $L^{2}\left(\mathbb{R}_{+}^{d}, d x\right)$ and was investigated in the one-dimensional, unweighted case by Stempak [St]. The interested reader should have no difficulties in formulating and proving proper statements.

Acknowledgements. The author is grateful to Professor Krzysztof Stempak for suggesting the topic and for many valuable remarks during the preparation of the paper.

\section{References}

[AW] R. Askey and S. Wainger, Mean convergence of expansions in Laguerre and Hermite series, Amer. J. Math. 87 (1965), 695-708.

[Di] U. Dinger, Weak type $(1,1)$ estimates of the maximal function for the Laguerre semigroup in finite dimensions, Rev. Mat. Iberoamericana 8 (1992), 93-120.

[D] J. Duoandikoetxea, Fourier Analysis, Amer. Math. Soc., Providence, 2001.

[GIT] C. E. Gutiérrez, A. Incognito and J. L. Torrea, Riesz transforms, g-functions, and multipliers for the Laguerre semigroup, Houston J. Math. 27 (2001), 579-592.

[GR] J. García-Cuerva and J. L. Rubio de Francia, Weighted Norm Inequalities and Related Topics, North-Holland Math. Stud. 116, North-Holland, Amsterdam, 1985.

[KeTh] R. Kerman and S. Thangavelu, Weighted norm inequalities for semigroups of operators and the norm convergence of Abel means of certain eigenfunction expansions, preprint.

[L] N. N. Lebedev, Special Functions and their Applications, Dover, New York, 1972.

[Mu1] B. Muckenhoupt, Poisson integrals for Hermite and Laguerre expansions, Trans. Amer. Math. Soc. 139 (1969), 231-242.

[Mu2] -, Mean convergence of Hermite and Laguerre series II, ibid. 147 (1970), 433-460. 
[S] E. M. Stein, Harmonic Analysis: Real-Variable Methods, Orthogonality, and Oscillatory Integrals, Princeton Univ. Press, Princeton, 1993.

[St] K. Stempak, Heat-diffusion and Poisson integrals for Laguerre expansions, Tôhoku Math. J. 46 (1994), 83-104.

[StTo] K. Stempak and J. L. Torrea, Poisson integrals and Riesz transforms for Hermite function expansions with weights, J. Funct. Anal., to appear.

[StZi] K. Stempak and J. Zienkiewicz, Twisted convolution and Riesz means, J. Anal. Math. 76 (1998), 93-107.

[Th1] S. Thangavelu, Lectures on Hermite and Laguerre Expansions, Math. Notes 42, Princeton Univ. Press, Princeton, 1993.

[Th2] - Harmonic Analysis on the Heisenberg Group, Birkhäuser, Boston, 1998.

Institute of Mathematics

Wrocław University of Technology

Wybrzeże Wyspiańskiego 27

50-370 Wrocław, Poland

E-mail: anowak@im.pwr.wroc.pl

Received July 12, 2002

Revised version March 8, 2003 